\title{
ESTUDIO MORFOLÓGICO DE SMILAX L. (SMILACACEAE) EN COSTA RICA, CON IMPLICACIONES SISTEMÁTICAS
}

\author{
LiLiAn FerRuFino ACOSTA \& JoRge GÓMEZ LAURITO \\ Escuela de Biología, Universidad de Costa Rica \\ 2060 San José, Costa Rica
}

\begin{abstract}
A morphologic revision of Costa Rican species of Smilax is presented. Traditionally, up to 14 species were accepted. In the present paper 7 species are recognized: Smilax domingensis, S. mollis, S. panamensis, S. spinosa, S. spissa, S. subpubescens, and S. vanilliodora. The following names are treated as synonyms: Smilax engleriana and $S$. kunthii of S. domingensis; S. hirsutior, S. angustiflora and S. candelariae of $S$. mollis, and $S$. chiriquensis and $S$. regelii var. albida of $S$. vanilliodora. Smilax regelii is excluded as a valid taxon and a lectotype of $S$. gymnopoda is designated. Dichotomous keys with vegetatives and reproductive characters (flowers and fruits) are presented, on the basis of field and herbaria observations. For all the species many important characters useful for identification were included, such as rhizome, stem, size of tepals and variation in berry colour during development stages.

RESUMEN. Se realizó una revisión morfológica de las especies de Smilax de Costa Rica. Tradicionalmente se han aceptado hasta 14 especies. En este trabajo se reconocen 7: Smilax domingensis, S. mollis, S. panamensis, S. spinosa, S. spissa, S. subpubescens y S. vanilliodora. Los siguientes nombres se tratan como sinónimos: Smilax engleriana y $S$. kunthii de $S$. domingensis; $S$. hirsutior, $S$. angustiflora y $S$. candelariae de $S$. mollis, y $S$. chiriquensis y $S$. regelii var. albida de $S$. vanilliodora. Smilax regelii se excluye como taxon válido y se designa un lectotipo de $S$. gymnopoda. Se elaboraron claves dicotómicas con características vegetativas y reproductivas (flores y frutos), con base en observaciones de campo y especímenes de herbario. En todas las especies se incluyeron diversos caracteres importantes para la identificación, como el rizoma, el tallo, el tamaño de los tépalos y la variación del color de las bayas a lo largo del desarrollo.
\end{abstract}

Palabras clave / Key words: Smilacaceae, Smilax, morfología, taxonomía, Costa Rica.

El género Smilax consta aproximadamente de 300 especies que habitan en zonas templadas y tropicales en ambos hemisferios (Judd et al. 2002). En Mesoamérica se han registrado al menos 25 especies. Standley (1937) comunica 14 especies en Costa Rica y Huft (1994) registra las siguientes 13 especies: Smilax angustiflora, S. candelariae, S. chiriquensis, S. domingensis, S. engleriana, S. hirsutior, S. kunthii, S. mollis, S. panamensis, S. spinosa, S. spissa, S. subpubescens y $S$. vanilliodora.

Durante mucho tiempo el género Smilax formó parte de la familia Liliaceae; pero en años recientes se ha incluido en su propia familia, Smilacaceae (Cronquist 1968, Dahlgren et al. 1985, Judd et al. 2002). Algunos estudios recientes argumentan la separación de Smilax de la familia Liliaceae, tales como: estudios de cromosomas (Vijayavalli \& Mathew 1990), composición de ácidos grasos en semillas (Morice 1970) y un estudio monográfico de las especies brasileñas (Andreata 1980). Tanto estudios morfológicos como análisis genéticos (ribulosa 1,5 bifosfato carboxilasa / oxigenasa, rbcL, y las regiones no codificadoras de ADN del cloroplasto, trn-L, trn-F) indican que la familia Smilacaceae es monofilética y pertenece al orden Liliales (Judd et al. 1999, 2002, Rudall et al. 2000).

En el neotrópico se han realizado estudios taxonómicos para tratar de distinguir las especies de este género. Gaskin \& Berry (1998) proponen una sinonimia en Smilax de la Guayana Venezolana y el uso de una combinación novedosa de caracteres, tales como: morfología de los tallos, bases de brotes laterales, presencia y ausencia de vainas aladas, articulación y longitud del pecíolo, morfología de las hojas, tipo de inflorescencia, longitud del pedicelo y del receptáculo, número de flores por receptáculo y 
características florales. Estos autores aceptan tres especies y reducen nueve nombres a sinonimia.

Guaglianone \& Gattuso (1991) realizan una revisión morfológica y un estudio anatómico de las estructuras vegetativas y reproductivas, así como de la distribución geográfica, la ecología y la fenología de Smilax en Argentina, donde describen e ilustran cinco especies. Andreata $(1980,1997)$ también realizó un estudio exomorfológico y anatómico y una revisión de Smilax en Brasil, abarcando 12 especies, junto con una historia taxonómica del género. Aunque se han considerado características diversas para distinguir las especies, los resultados no han sido los esperados.

Desde la época precolombina la raíz de cuculmeca y zarzaparrilla, nombres populares de ciertas especies de Smilax en Centro América, ha sido considerada en la medicina popular contra problemas diuréticos, infecciones dermatológicas, desórdenes gastrointestinales, reumatismo, vaginitis, como anticonceptivo, para regulación menstrual, anemia, mordeduras de serpientes y artritis (Ocampo 1994, Gupta 1995). A pesar de que en la región mesoamericana se han realizado investigaciones fitoquímicas y etnobotánicas, no se sabe con certeza cuáles son las especies utilizadas, lo que pone en evidencia una gran incertidumbre en la información disponible.

El objetivo principal de este trabajo fue mejorar las descripciones existentes y resolver problemas taxonómicos y de sinonimia en las especies de Costa Rica, lo que facilitará la identificación de las especies registradas en la región y zonas aledañas. Además, este estudio comprende un análisis morfológico de las estructuras vegetativas y reproductivas, teniendo en cuenta la variabilidad morfológica de las hojas y el color de los frutos, que son caracteres que han sido observados en la naturaleza y en especímenes de herbario.

\section{Historia taxonómica de Smilacaceae y Smilax}

\section{Familia Smilacaceae Ventenat}

La familia Smilacaceae está compuesta por los géneros Smilax L. (300 spp.) y Heterosmilax Kunth (11 spp.), que se distribuyen en regiones templadas, tropicales y subtropicales (Heywood 1978, Dahlgren et al. 1985).

Este taxon fue descrito por Ventenat (1799) con base en el género Smilax L. Este concepto fue seguido por diversos autores (Endlicher 1836, Lindley 1836, Grisebach 1842, Kunth 1850, De Candolle 1878). Otros autores (Bentham \& Hooker 1880, Engler 1888, Melchior 1964) tratan Smilax en la familia Liliaceae. Engler (1888) y Melchior (1964) dividen Liliaceae en 13 subfamilias; entre éstas Smilacoideae, que se compone de cuatro géneros: Rhipogonum Forst. \& Forst. (variante ortográfica en la literatura: Ripogonum), Smilax, Pseudosmilax Hayata y Heterosmilax. Dahlgren et al. (1985) incluyen estos cuatro géneros en la familia Smilacaceae, que dividen en dos subfamilias: Smilacoideae, con tres géneros, y Rhipogonoideae con el género Rhipogonum.

Además de Liliaceae o Smilacaceae, la familia se ha incluido en Liliiflorae (Melchior 1964), Liliales (Hutchinson 1934, Cronquist 1968, Heywood 1978, Goldberg 1989, Judd et al. 1999, 2002), Asparagales (Dahlgren \& Clifford 1982), Dioscoreales (Dahlgren et al. 1985) y Smilacales (Takhtajan 1969). Dahlgren et al. (1985) comentan que la familia puede ser considerada junto a Petermanniaceae dentro de Dioscoreales. Judd et al. (1999, 2002) incluyen en la familia Smilax y Rhipogonum, mientras que Thorne (1992) considera que Smilacaceae es una familia monogenérica.

Otra clasificación de Smilacaceae es la que incluye tres géneros: Rhipogonum, Smilax y Heterosmilax, apoyada en gran parte por Melchior (1964), Heywood (1978), Koyama (1983), Dahlgren et al. (1985) y Andreata (1997). Pseudosmilax se reduce a sinónimo de Heterosmilax (Koyama 1984).

Heterosmilax se distribuye en el sudeste de Asia. Este es un género muy próximo a Smilax, que se diferencia de éste por el perigonio soldado (urceolado) y tres estambres (raramente 9-12) generalmente unidos.

Smilax es el género con mayor importancia económica, distribuido en regiones templadas, tropicales y subtropicales en ambos hemisferios. Se caracteriza por un perigonio libre, seis estambres libres y anteras confluentes (Koyama 1983, Dahlgren et al. 1985, Huft 1994).

Otro factor que ha causado polémica es la posición 
filogenética de Smilacaceae en relación con otras monocotiledóneas. Cronquist (1981) considera que Smilacaceae y Dioscoreaceae son las familias más especializadas de Liliales, mientras que Dahlgren et al. (1985) consideran que Smilacaceae es una de las seis familias no basales de las monocotiledóneas. Ellos sostienen que Dioscoreaceae está muy relacionada con Smilacaceae y ésta última con Petermanniaceae y Liliaceae. Tanto estudios morfológicos (Conran 1989) como de secuencias de ADN (Chase et al. 1995a, 1995b, 2000, Judd et al. 1999, 2002, Rudall et al. 2000, Patterson \& Givnish 2002) favorecen la ubicación de Smilacaceae en el orden Liliales.

\section{El género Smilax L.}

Tournefort (1694, citado por Arveiller 1985) fue el primer taxónomo que estudió el género Smilax; él consideró importante el color de los frutos.

Linneo (1753) describe una planta de tallos angulosos con aguijones y hojas dentado-crenadas con el nombre Smilax aspera. Según Vandercolme (1947) el nombre Smilax se deriva del griego smile que significa "raspa", por la presencia de acúleos que aparecen en la mayoría de las especies. Pero otros autores opinan que Smilax significa hierba para atar y que el nombre fue usado por primera vez por Plinio para referirse a una hierba de atar, que es Smilax aspera. Este término también se usó para referirse a las especies de avena y a los árboles de tejo (Quattrocchi 2000).

Linneo (1753) coloca a Smilax en la clase XXII Dioecia y el orden VI Hexandria, entre los géneros Tamus L. y Cissampelos L. Ventenat (1799) 1o incluye en el orden III y lo considera dentro de Smilacaceae, haciendo referencia a los aspectos vegetativos y florales. Poiret (1804) es seguidor del sistema de Linneo; presenta una descripción morfológica de Smilax y lo ubica entre los géneros Salpiglossis Ruiz \& Pav. y Tragopogon L., ambos en la subclase Dicotyledoneae. El mismo Poiret (1823) después incluye a Smilax entre Tamus y Dioscorea L.

Humboldt \& Bonpland (1815) realizan un viaje a América del Sur y encuentran 12 especies nuevas de Smilax. Colocan a Smilax entre Tamus y Dioscorea, y a Asparagea Juss. entre Dianella Lam. y Dioscorea.
Endlicher (1836) sitúa Smilax en su propio orden LVI Smilacaceae.

Lindley (1836) incluye Smilax en el grupo IV Retosae y en el orden CCLVI Smilacaceae. Este autor sitúa Smilax en la clase V Distyogenes y el orden LXIX Smilaceae, al lado del género Rhipogonum.

Grisebach (1842) realiza el tratamiento de 33 especies de Smilax; divide el género en Pharmacosmilax y Pachysmilax por la base y la forma de la antera, y la consistencia y el patrón de la nervadura de la hoja. Ubica Smilax en Smilacaceae, cerca de Herreria Ruiz \& Pav.

Kunth (1850) enumera 188 especies de Smilax y estudia 124. Ubica a Smilax en Smilaceae.

Vandercolme (1871-73) estudia las especies medicinales de Smilax e incluye $S$. officinalis Humb. \& Kunth, S. sarsaparrilla L. y S. china L. como plantas de uso medicinal.

De Candolle (1878) presenta una monografía de Smilacaceae con información valiosa sobre las partes vegetativas y florales, distribución geográfica, clave analítica, descripciones completas y un análisis de polen de las especies. Divide el género en cuatro grupos: Nermexia, Coilanthus, Eusmilax y Pleiosmilax. Incluye las especies de Costa Rica en Eusmilax y describe tres especies nuevas del país: Smilax angustiflora, S. candelariae y S. subpubescens. Ubica a Smilax entre Heterosmilax y Rhipogonum. Esta última idea fue apoyada por Bentham \& Hooker (1880) y Engler (1888).

Morong (1894) en su trabajo de las Smilaceae de Norte y Centro América, describe Smilax panamensis, una nueva especie de Panamá. Baillon (1894) coloca Smilax, Heterosmilax y Rhipogonum en la familia Smilaceae, próxima a Stemoneae y Herrerieae.

Killip \& Morton (1936) realizan una revisión de las especies de Smilax de Centro América y México y reconocen nueve especies nuevas. Takhtajan (1969) considera Smilax y Heterosmilax dentro de la subfamilia Smilacoideae y Rhipogonum en Rhipogonoideae, ambas pertenecientes a Smilacaceae y al orden Smilacales.

En los últimos 40 años, el género ha sido estudiado por Koyama en Asia (1960, 1974, 1975, 1977), quien ha agregado nuevos taxa de la región, ha hecho claves para la identificación de grupos y 
especies, y ha ilustrado y colaborado en floras locales.

Dahlgren \& Clifford (1982) incluyen Smilax, Heterosmilax, Pseudosmilax y Rhipogonum en Smilacaceae. Dahlgren et al. (1985) y Dahlgren (1989) elevan el género Rhipogonum a la categoría de familia: Rhipogonaceae.

En Brasil, Andreata (1979, 1980, 1982, 1984a) estudia 12 especies de Smilax y elabora la primera clave para el reconocimiento de los táxones. Ella (1997) realiza una revisión taxonómica de las especies de Brasil y hace una descripción completa, ilustra, resuelve problemas taxonómicos y de tipificación y amplía los datos de distribución geográfica. Además, sus contribuciones comprenden los trabajos de las floras regionales de Brasil (Andreata \& Wanderley 1984b, Andreata \& Cowley 1987, Andreata 1995, 1996).

Guaglianone \& Gattuso (1991) revisan cinco especies del género en Argentina: Smilax assumptionis A. DC., S. campestris Griseb, S. cognata Kunth, S. fluminensis Steud. y S. pilcomayensis Guaglian. \& Gattuso (especie nueva). También elaboran una clave basada en características exomorfológicas e histológicas, haciendo énfasis en anatomía, nectarios florales y compuestos químicos.

Huft (1994) realiza el tratamiento sistemático del género para la flora mesoamericana. Hace una descripción botánica y agrega una clave dicotómica. Huft (2001) también hace el tratamiento del género en la Flora de Nicaragua, que abarca 12 especies.

Judd et al. (1999, 2002) consideran Smilacaceae en Liliales, con Smilax y Rhipogonum. La mayoría de los autores concuerdan en que Smilax pertenece a Smilacaceae y está relacionado con los miembros de Liliales.

\section{Material y métodos}

Para caracterizar el rizoma se observó el color y se determinó si el rizoma presentaba: a) engrosamiento tuberoso, y b) entrenudos engrosados.

También se determinó la forma y el color de los aguijones en tallos y hojas. La longitud y el ancho de las hojas se midieron en varias hojas de cada especie.

Se midió el largo del pecíolo en las hojas. Además, se midió y observó el color de la vaina en plantas observadas en el campo. Se determinó la dis- posición de las inflorescencias en ramas y la disposición y el número de flores en la inflorescencia. Se recolectaron por lo menos dos muestras de las plantas en floración. Se midió bajo un estereoscopio el tamaño de tépalos, estambres (filamento y antera) y pistilo (estilo y estigma). El diámetro de los frutos fue medido; además se observó el color y la forma de los frutos durante la maduración. Se contó el número de los frutos por lo menos en tres infrutescencias de cada planta. Se contó el número de semillas en cuatro a seis frutos.

Se extrajeron muestras de polen de ejemplares depositados en herbarios. Cada muestra se colocó sobre una cinta de carbón adhesiva (por ambas caras). Después se montó sobre una base de aluminio, en seguida se cubrió con $30 \mathrm{~nm}$ de oro utilizando un cobertor iónico (Eiko IB-3, Japón). Posteriormente cada muestra fue observada con un microscopio electrónico de barrido S-570, utilizando un voltaje de aceleración de $25 \mathrm{KV}$. En la toma de micrografías se utilizó la película Verichrome Pan 120 (Kodak).

Se examinó material de los siguientes herbarios: Herbario Nacional de Costa Rica (CR), Herbario de la Universidad de Costa Rica (USJ), Instituto Nacional de Biodiversidad (INB), Herbario Juvenal Valerio de la Universidad Nacional (JVR), Missouri Botanical Garden (MO), Universidad de Panamá (PMA) e Instituto de Investigación Tropical Smithsonian (STRI). También se consultaron bases de datos en Internet: W3 TROPICOS del Jardín Botánico de Missouri, el Índice Internacional de Nombres de Plantas (IPNI); además, un índice de publicaciones botánicas, otro índice de botánicos, el índice de especímenes botánicos del Herbario Gray de la Universidad de Harvard $(\mathrm{GH})$ y fotografías de tipos del Herbario del Field Museum de Chicago (F).

\section{Resultados y discusión}

\section{Morfología}

\section{HÁBITO Y SISTEMA REPRODUCTIVO}

Las especies del género Smilax en Costa Rica son bejucos leñosos o herbáceos, dioicos, de pequeña y mediana longitud. Algunas plantas podrían alcanzar 20 o 30 m de largo, según el soporte. 


\section{RIZOMA}

En Costa Rica no existen trabajos sobre la organografía del rizoma de Smilax. Según la morfología externa, el sistema subterráneo se divide en dos tipos: a) con engrosamiento tuberoso (Fig. 2 ), b) con nudos y entrenudos engrosados (Fig. 4). Smilax domingensis, S. panamensis y S. spissa presentan el primer tipo, que es un rizoma formado por una parte caulinar, de consistencia leñosa. El sistema caulinar subterráneo está cubierto por catafilos dispuestos dísticamente y raíces adventicias, que facilitan el enraizamiento. El color del rizoma varía según las condiciones ambientales.

Smilax spinosa, S. vanilliodora y $S$. mollis presentan el segundo tipo, que es un rizoma con pequeños engrosamientos en los nudos y entrenudos y las raíces adventicias pueden ser cilíndricas o cuadrangulares como es el caso de $S$. vanilliodora.

\section{TALLOS}

Los tallos son cilíndricos en la mayoría de las especies (Fig 2); en S. vanilliodora son cuadrangulares, con o sin alas (Fig 11). La superficie puede ser estriada, como en S. spinosa, o lisa, como en la mayoría de las especies.

Se halla pubescencia en $S$. mollis y $S$. subpubescens, especialmente en estructuras jóvenes de la planta. Los aguijones pueden ser grandes o pequeños. En $S$. panamensis son grandes y robustos en la parte inferior del tallo; en $S$. domingensis el tamaño depende de la edad del bejuco y en $S$. vanilliodora son aplanados. Dos especies no presentan aguijones: S. mollis y $S$. subpubescens.

El color del tallo varía según el ambiente donde crezca la planta. En $S$. domingensis puede ser rojo a morado en la parte inferior; en la mayoría de las especies es verde.

\section{HOJAS}

La forma y el tamaño de las hojas varían entre los bejucos de una misma especie, aunque muchos autores utilizan estos caracteres para distinguir las especies de Smilax. Esta variación ha sido interpretada como respuesta a los factores ambientales; se trata de una plasticidad fenotípica (Andreata 1992) de origen genético o de polimorfismo foliar como es el caso de Smilax aspera (Vernet 1962).
Todas las especies presentan hojas simples, alternas, glabras o pubescentes, con un pecíolo bien diferenciado. El pecíolo puede ser recurvado o acanalado, con o sin acúleos y con dos zarcillos.

Los zarcillos son cilíndricos, robustos o herbáceos. Andreata (1997) menciona que las especies arbustivas o subarbustivas carecen de zarcillos. Diversas interpretaciones del origen de los zarcillos han sido resumidas por Domin (1911), y discutidas por Arber (1920), Clos (1857) y Glück (1901). Estos autores consideran que los zarcillos se originan de divisiones longitudinales congénitas del pecíolo (corisis).

La vaina puede ser persistente en algunas especies o caduca en otras. En $S$. panamensis es persistente y prominente. Smilax subpubescens presenta un tomento amarillento persistente en la base del pecíolo.

La lámina puede ser pubescente, como en $S$. mollis y en las hojas jóvenes o base del pecíolo de $S$. subpubescens. Las demás especies costarricenses presentan hojas glabras. La lámina es coriácea o membranácea en la mayoría de los casos; es coriácea en algunos bejucos de $S$. domingensis.

La nervadura de la lámina es acródroma y varía de 5 a 7 ó 7 a 9 nervios principales según la especie, con nervaduras reticuladas entre las principales, en la mayoría de las especies, a excepción de $S$. spissa que presenta nervaduras paralelas (Fig. 8).

\section{INFLORESCENCIAS}

\subsection{Inflorescencias estaminadas}

La mayoría de autores considera que las inflorescencias de Smilax son umbelas, a excepción de De Candolle (1878) y Andreata (1980), que las interpretan como cimas umbeliformes.

Este trabajo muestra que cada inflorescencia parcial es un racimo o una umbela solitaria (Fig. 2 y 5).

El androceo está formado por seis estambres libres, alternando con los tépalos. Las anteras son oblongas, bitecas, basifijas, introrsas, con dehiscencia longitudinal. El tamaño de la antera varía en cada flor y umbela. Los filamentos son más largos que las anteras en la mayoría de las especies.

La morfología del androceo en Smilax ha sido interpretada de diversas maneras. De Candolle (1878) y otros autores hacen una descripción de anteras confluentes, bitecas o monotecas. Cronquist (1981) describe las anteras tetrasporangiadas, bitecas y 

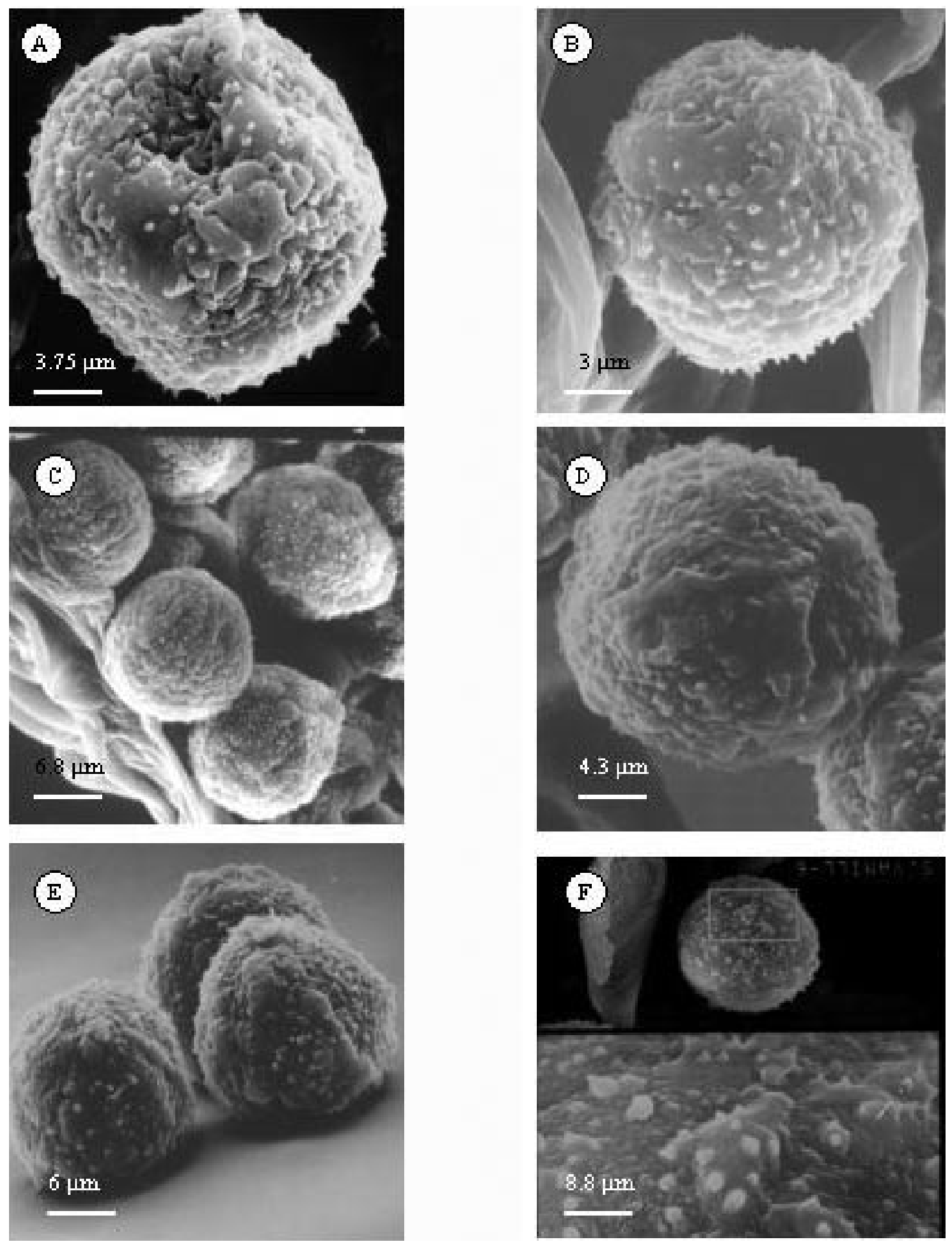

Figura 1. Granos de polen de Smilax. A, B, C, D, E. Vista general; F. Detalle de la exina con gránulos y espínulas. A. S. domingensis; B. S. mollis; C. S. spinosa; D y F. S. subpubescens; E. S. vanilliodora. 
uniloculares. Andreata (1997) las describe con tecas biesporangiadas, con o sin apículo, muchas veces dehiscentes, de forma oblonga, elíptica o linear.

\subsubsection{Polen}

Los primeros trabajos sobre la morfología del polen de Smilax iniciaron con De Candolle (1878) y Erdtman (1966). Andreata (1980) analiza seis especies de Brasil, utilizando microscopía electrónica. y lo considera pequeño (18.5-21.1 mm), apolar, inaperturado, esferoidal y espiculado. Roubik \& Moreno (1991) estudiaron cinco especies de la Isla de Barro Colorado en Panamá y elaboraron una clave usando el tamaño de los granos, desplegamiento de exina y conspicuidad verrucosa. Furness \& Rudall (1999) estudian el polen de $S$. hispida Muhl. ex Torr. y S. sieboldii Miq. y lo describen como inaperturado, esferoidal, con gránulos sobre la superficie y espínulas que varían en forma.

En el presente estudio se analizaron cinco especies de Smilax registradas en Costa Rica, las que presentan granos de polen que varían entre 15 y 21 micras, son subglobosos, granulosos y espinosos. El tamaño varía entre plantas de la misma especie (Fig 1).

\section{2 Inflorescencias pistiladas}

Las especies de Smilax de Costa Rica presentan seis tépalos, la mayoría de $4 \mathrm{~mm}$, excepto en $S$. spinosa $(2 \mathrm{~mm})$, son blancos o crema con tres estaminodios oblongos a filiformes. Los estaminodios están constituidos por tejido de origen epidérmico, considerados estructuras vestigiales (Andreata 1997).

El gineceo es sincárpico, tricarpelar, trilocular, con un óvulo en cada lóculo; es bitégmico, con placentación axilar, ovario ortótropo, súpero, glabro, subgloboso, con 3 estigmas, libres o parcialmente unidos, apicales, secos y papilosos.

\section{FRUTOS}

Los frutos son bayas globosas, que varían de tamaño entre los bejucos de la misma especie. El exocarpo es de color morado o rojo en Smilax domingensis, S. spissa y $S$. spinosa. En $S$. vanilliodora es rojo y en $S$. panamensis, $S$. mollis y $S$. subpubescens es anaranjado. Las especies de Smilax presentan tres fases de coloración: una inicial que es verde, una intermedia, que puede ser roja como en $S$. domingensis y crema en $S$. panamensis, y la fase final con el color de la maduración: rojo, morado, negro y anaranjado.
El número de frutos varía según la infrutescencia. Las semillas son esféricas y varían de 1 a 3 por baya.

\section{Tratamiento sistemático}

Smilax L., Sp. Pl. ed. 2: 1028. 1753; Gen. Pl. ed. 455. 1754;

Parillax Raf., Medical Fl. U.S. 2264. 1828.

OTRAS REFERENCIAS BIBLIOGRÁFICAS: Duham, Traité Arbr. Arbust. 1: 233. 1801; Poir., Encycl. Met. Bot. 6: 464. 1804; Endl., Gen. Pl. 1184. 1836; Griseb. in Mart., Fl. Bras. 3(1): 3. 1842; Torrey, Fl. New York 2: 302. 1843; Kunth, Enum. Pl. 5: 160. 1850; A. DC. in A. \& C. DC., Monogr. Phan. 1: 45. 1878; Benth., Fl. Austral. 7: 6. 1878; Benth. \& Hook., Gen. Pl. 3(1): 763. 1880; Engler, Nat. Pflanzen. 2(5): 88. 1888; Hook., Fl. Brit. Ind. 6: 302. 1892; Morong, Bull. Torrey Bot. Club 21(10): 420. 1894; Vander., Adansonia 10: 74. 187173; Koyama, Fl. Taiwan 5: 110. 1979; Andreata, Arq. Jard. Bot. Rio de Janeiro 24: 179. 1980; Koyama, Fl. Cambodge, Laos et Viêt-Nam 20: 69. 1983; Andreata, Hoehnea 11: 114. 1984; Guaglianone \& Gattuso, Bol. Soc. Argent. Bot. 27(1-2): 105. 1991; Andreata, Pesquisas, Bot. 47: 1-243. 1997.

TIPO DEL GÉNERO: Smilax aspera L. (cf. Britton \& Brown 1913) (IDC microficha 695.1182.6.II.7)

Bejucos subleñosos, rara vez herbáceos, dioicos, que se originan de un rizoma delgado o grueso. Tallos redondos o cuadrados, pubescentes o glabros, que usualmente trepan mediante pares de zarcillos que se originan en los pecíolos y a menudo poseen aguijones en tallos. Hojas alternas, con 5 a 9 nervios paralelos, o triplinervias, con el par interior saliendo un poco arriba de la base y conectadas por nervaduras reticuladas (paralelas en S. spissa), láminas coriáceas, cartáceas o membranáceas. Inflorescencia dispuesta en umbelas axilares, a veces racemosas, pedúnculo cilíndrico o subcilíndrico. Flores estaminadas, pequeñas, blancas, con 6 tépalos libres, iguales, estambres 6 , libres, anteras bitecas, basifijas, más largas o más cortas que los filamentos, polen granuloso-espinoso. Flores pistiladas pequeñas, blancas, con ovario súpero, tricarpelar, 1 ó 2 óvulos en cada lóculo, estaminodios presentes. Fruto una baya globosa, negra, púrpura, roja o anaranjada, con 1 a 3 semillas rojizas o negras (Cronquist 1981, Watson \& Dallwitz 1992, Huft 1994, Andreata 1997). 


\section{CLAVE DICOTÓMICA CON CARACTERES VEGETATIVOS DE SMILAX DE COSTA RICA}

1. Plantas pubescentes o casi glabras en la madurez, sin aguijones en el tallo. 2

2. Ramas obtusamente cuadrangulares, glabras en la madurez o a veces con poco tomento rojo en la base de los pecíolos

S. subpubescens

2. Ramas cilíndricas, variadamente pilosas, pubescencia tomentosa ............................................................ S. mollis

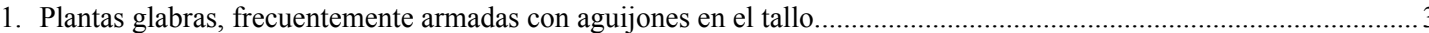

3. Tallos cuadrangulares, casi siempre con alas ................................................................................... S. vanilliodora

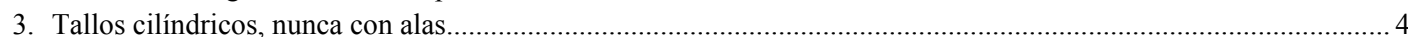

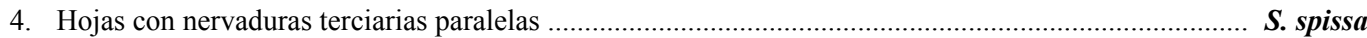

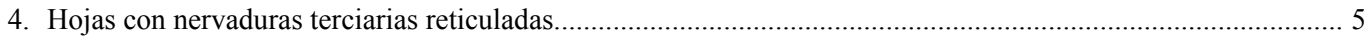

5. Tallos superiores en zigzag, hojas con 5 nervios desde la base; rizoma con entrenudos engrosados

S. spinosa

5. Tallos superiores casi rectos, hojas con 5 a 9 nervios desde la base; rizoma tuberoso...................................... 6 6. Hojas con 5-7 nervios desde la base, rizoma rojo; tallos inferiores de color rojo o morado...... S. domingensis 6. Hojas con 7-9 nervios desde la base; rizoma blanco; tallos inferiores de color verde............ S. panamensis

\section{CLAVE DICOTÓMICA PARA ESPECÍMENES CON FLORES ESTAMINADAS Y PISTILADAS DE SMILAX DE COSTA RICA}

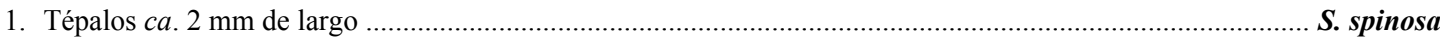

1. Tépalos $c a$. 3.5-6 $\mathrm{mm}$ de largo 2

2. Plantas pubescentes, glabras en la madurez, a veces con poca pubescencia en la base de los pecíolos, sin aguijones en el tallo. 3

3. Ramas obtusamente cuadrangulares, glabras en la madurez o a veces con poco tomentoso rojo en la base de los pecíolos

S. subpubescens

3. Ramas cilíndricas, variadamente pubescentes

S. mollis

2. Plantas glabras, armadas con aguijones en el tallo.

4. Tallos cuadrangulares, casi siempre con alas

S. vanilliodora

4. Tallos cilíndricos, nunca con alas.

S. domingensis

5. Pedúnculos más cortos que el pecíolo subyacente

5. Pedúnculos más largos que el pecíolo subyacente, umbelas en racimos o solitarias

6. Hojas con 7 nervios desde la base, conectados por nervaduras reticuladas, anteras más largas que los filamentos

S. panamensis

6. Hojas con 5 nervios desde la base, conectados por nervaduras paralelas, anteras más cortas que los filamentos

S. spissa

\section{CLAVE DICOTÓMICA PARA ESPECÍMENES CON FRUTOS DE SMILAX DE COSTA RICA}

1. Plantas pubescentes o casi glabras en la madurez, sin aguijones.

2

2. Ramas obtusamente cuadrangulares, glabras en la madurez o a veces con poco tomento rojo en la base de los pecíolos; frutos anaranjado brillante; pedúnculo aplanado

S. subpubescens

2. Ramas cilíndricas, variadamente pubescentes, tomentosas; frutos anaranjados; pedúnculo cilíndrico......... $\boldsymbol{S}$. mollis

1. Plantas glabras, frecuentemente armadas con aguijones

3. Tallos cuadrangulares, casi siempre con alas; frutos rojos o negros ................................................. S. vanilliodora

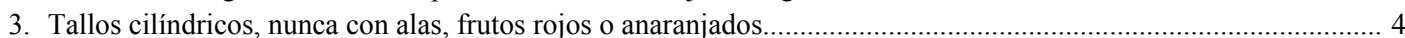

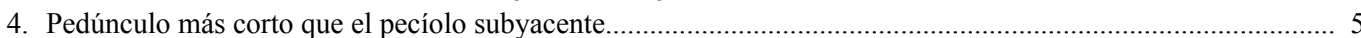

5. Tallos superiores en zigzag; hojas con 5 nervios desde la base ....................................................... S. spinosa

5. Tallos superiores casi rectos; hojas con 5-7 nervios desde la base............................................ S. domingensis

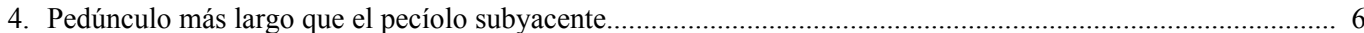

6. Frutos anaranjados; hojas con 7 nervios desde la base, conectados por nervaduras reticuladas; brácteas florales persistentes

S. panamensis

6. Frutos rojos; hojas con 5 nervios desde la base, conectados por nervaduras paralelas; brácteas florales no persistentes 
1. Smilax domingensis Willd., Sp. Pl. 4: 783. 1806. TIPO: Santo Domingo. Porto Rico. Richard s.n. (holotipo B, IDC microficha 7440.1338. II.2; IDC microficha 7440.1338.II.3).

FIG. 2

Smilax schlechtendalii Kunth, Enum. P1. 5: 224. 1850. TIPO: México. In sylvis Misantlae et prope Chiconquiaco, Schiede \& Deppe s.n. (holotipo, B; isotipo, $\mathrm{K}, \mathrm{MO}$ !, W).

Smilax engleriana Apt, Repert. Spec. Nov. Regni Veg. 18: 407. 1922. TIPO: Costa Rica. Santa Rosa del Copey, alt. 1100 m, Tonduz 11732 (lectotipo designado por Killip y Morton, Publ. Carnegie Inst. Wash. 461: 266. 1936, B; isolectotipos, BM, CR!, US!, W); syn. nov.

Smilax microscola (B. L. Rob.) Killip \& C. Morton, Publ. Carnegie Inst. Wash. 461: 267. 1936. Smilax domingensis var. microscola B. L. Rob., Proc. Amer. Acad. Arts 35(16): 323. 1900. TIPO: México. Chiapas: between Tumbla and El Salto, 29 Oct 1895, E.W. Nelson 3392 (holotipo, GH!; isotipo, US!).

Smilax kunthii Killip \& Morton, Publ. Carnegie Inst. Wash. 461: 269. 1936. Smilax floribunda Kunth, Enum. Pl. 5:229. 1850. TIPO: Ecuador. Ruiz \& Pavón s.n. \& Hartweg s.n. (Sintipo B, foto $10062 \mathrm{~F}$ !).

Smilax caudata Lundell, Wrightia 3(8): 162. 1966. TIPO: Guatemala. Alta Verapaz: on SebolCoban Rd., between km 285-286, between Chiraete and Chapultepec Farm, in high forest, 24 May 1964, E. Contreras 4783 (holotipo, LL; isotipo, MO!).

Rizoma con engrosamiento tuberoso, rojo o morado; escamas coriáceas, persistentes; raíces cilíndricas con escamas coriáceas, caducas. Tallos cilíndricos, glabros, armados con aguijones recurvados en la parte inferior, inerme en la parte superior, rojos en la base. Hojas ovadas a lanceoladas, membranáceas a coriáceas o cartáceas, $5.5-13 \times 2-8.7 \mathrm{~cm} ; 5-7$ nervios desde la base conectados por nervaduras reticuladas, conspicuas; ápice acuminado o brevicuspidado, base aguda, a veces redondeada, margen entero, ocasionalmente hojas nuevas rojas; pecíolos $0.7-2 \mathrm{~cm}$ de largo. Umbelas solitarias, las estaminadas con pedúnculo cilíndrico o subcilíndrico, $0.1-1 \mathrm{~cm}$, más corto que el pecíolo subyacente; tépalos $3.5-7 \mathrm{~mm}$, filamentos 2$3 \mathrm{~mm}$, anteras 1-2 mm, más cortas que los filamentos; las pistiladas con pedúnculo cilíndrico o subcilíndrico, 0-1.2 cm; tépalos 4-5 mm. Bayas 6-10 mm de diámetro, rojas, moradas o negras.

DISTRIBUCIÓN Y HÁBITAT. Smilax domingensis es una especie abundante que se encuentra distribuida en bosques húmedos, montanos y premontanos a una elevación de 0-1800 m (Fig. 3). Se distribuye desde Veracruz y Oaxaca hasta Panamá y las Antillas.

NOMBRES COMUNES Y USOS. Cuculmeca roja, cuculmeca morada, bejuco de membrillo, corona de Cristo, raíz de zarzaparrilla, espino de corona (Nelson 1985). El rizoma se usa como depurativo, diurético, antianémico, vigorizador, sudorífico, antirreumático (Núñez 1982, Ocampo 1997), antiblenorrágico, antiherpético y para otras enfermedades de la piel (Núñez 1982). En Honduras es usado para hacer canastas (Nelson 1985).

Smilax domingensis se caracteriza por el rizoma tuberoso rojo o morado, tallos cilíndricos con aguijones, las hojas jóvenes rojas, pedúnculos más cortos que el pecíolo subyacente, tépalos $c a .4 \mathrm{~mm}$, bayas rojas o moradas.

Es una especie muy variable y de distribución amplia. Los nombres que se consideran sinónimos se

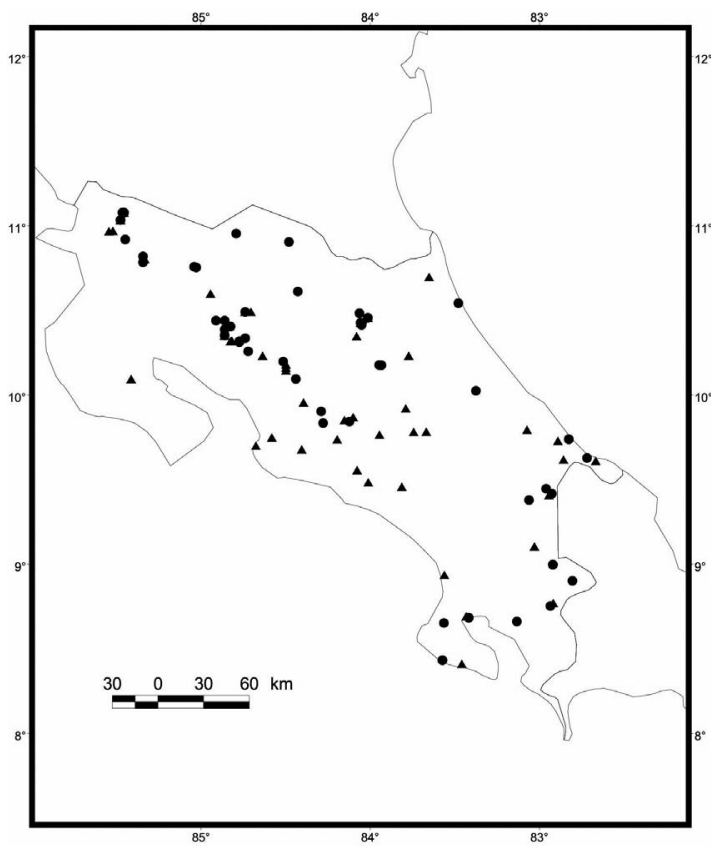

Figura 3. Distribución geográfica de Smilax domingensis (•) y $S$. mollis $(\boldsymbol{\Delta})$ en Costa Rica. 


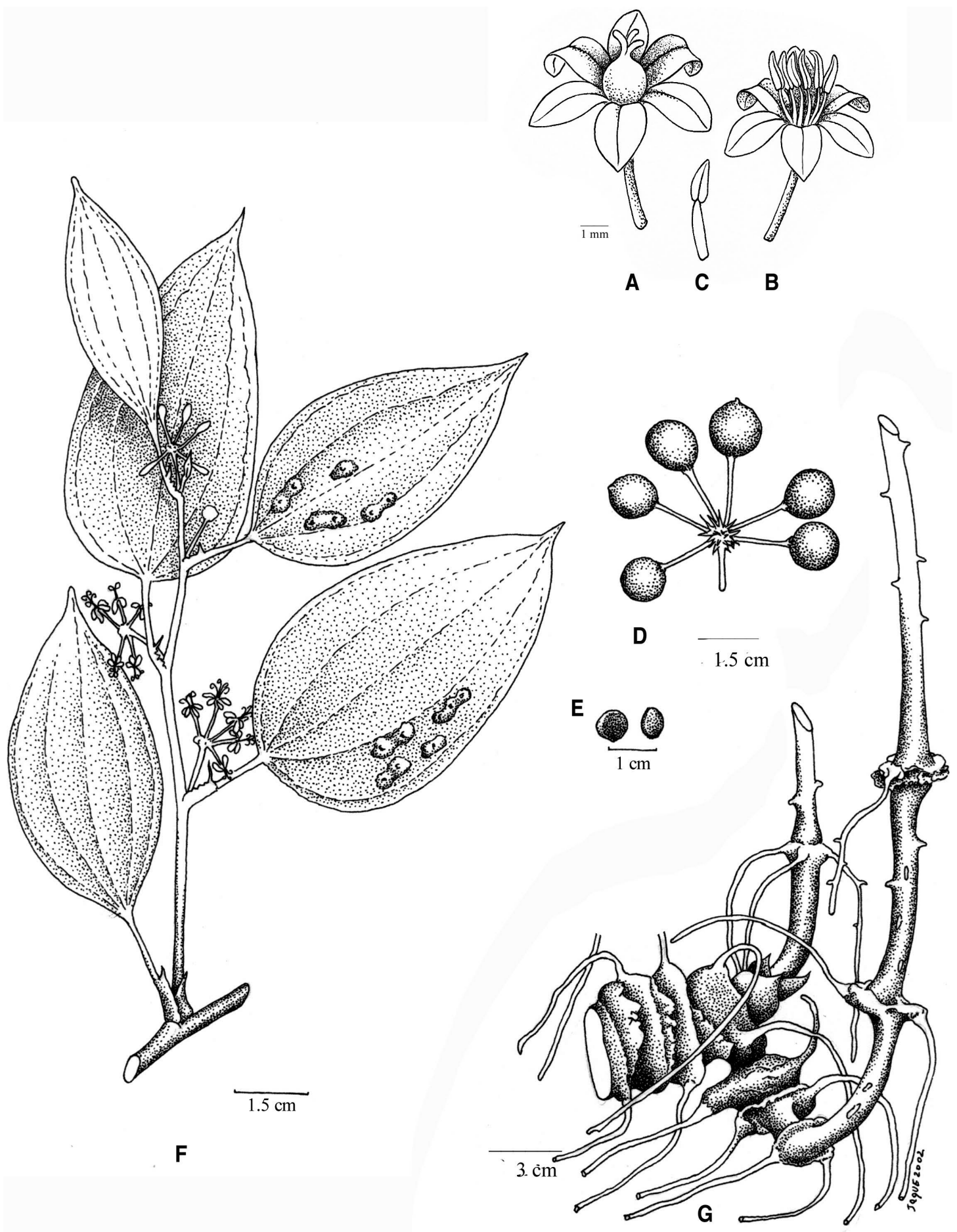

Figura 2. Smilax domingensis Willd.: A. Flor femenina, B. Flor masculina, C. Estambre, D. Frutos, E. Semillas, F. Rama fértil con agallas y flores, G. Tallo y rizoma. 
le han dado a especímenes que presentan variaciones morfológicas en la textura de las hojas y el color de los tallos. Apt (1922) describe S. engleriana y la diferencia de $S$. domingensis por sus hojas coriáceas, las nervaduras principales conspicuas e impresas en el haz, conectadas por nervaduras inconspicuas. Sin embargo, en la descripción no hace mención de los frutos.

La plasticidad fenotípica de Smilax, en particular de la parte vegetativa, ha sido ya discutida por muchos investigadores. Andreata (1980) y Andreata \& Pereira (1990) realizan una revisión de las especies brasileñas de Smilax y encuentran una gran variación entre las hojas juveniles y las hojas adultas, tanto en la forma como en su consistencia. La lámina foliar se torna más gruesa, gradualmente a lo largo del desarrollo de las hojas, es generalmente membranácea en las hojas juveniles, pasando de papirácea a coriácea. Además, estos autores mencionan que algunos caracteres como el tamaño de los entrenudos, la longitud del pecíolo, la forma del limbo, el tipo de base, el ápice y las nervaduras del limbo, son relevantes desde el punto de vista taxonómico si se usan en conjunto, para la fase de plántula o plantas jóvenes.

Esta variabilidad morfológica es discutida por Ellen-MacDonald \& Chinnappa (1988), quienes sugieren que ésta se debe a una variación de cariotipos. Algunas de estas expresiones en la variación probablemente representan frecuencias de genes fijados en algunas poblaciones. Sin embargo, aún no se conocen estudios de Smilax que apoyen estas hipótesis.

Otro factor que juega un papel importante son las condiciones ambientales que varían entre los diferentes microhábitats. Ferrufino (datos inéditos) realiza un estudio de fenología de cinco especies de Smilax en tres sitios de Costa Rica y concluye que la luz y la precipitación son factores que influyen en la producción de hojas, flores y frutos. Además, observa que los bejucos que crecen en lugares abiertos tienen hojas más largas y anchas en comparación con las plantas que crecen en zonas sombreadas.

Huft (1994) describe S. kunthii con base en material de Centroamérica. He identificado el material examinado por este autor, y las colecciones de Williams \& Molina 11775, Rohweder 989, Stevens 15107 y Hammel 6054 (todos en MO) son especímenes en floración de $S$. domingensis. El tamaño y el color de los frutos están basados en Antonio 715 (MO), identificado como S. subpubescens.

En el Field Museum de Chicago se encuentra una foto (\# 10062) que al parecer es el tipo de Smilax floribunda Kunth, recolectado en Perú por Ruiz, que posee características morfológicas de $S$. domingensis. En la descripción, Kunth (1850) hace énfasis en dos especímenes recolectados por Hartweg y Ruiz \& Pavón en Perú. Sin embargo, Killip \& Morton (1936) hacen mención del espécimen recolectado por Hartweg; y rectifican que el tipo de esta especie fue recolectado en Ecuador ("Perú es un error"). Además, esta sinonimia es apoyada por la descripción de $S$. floribunda que coincide con la caracterísiticas morfológicas de $S$. domingensis.

ESPECÍMENES EXAMINADOS. BELICE. Corozal: $1 \mathrm{mi}$ west of northern highway on secondary road, $1 \mathrm{mi}$ north of Buena Vista, 100 ft., 23 Jun 1973 (fr), Croat 24962 (MO). COLOMBIA. Valle: El Cairo, Cerro del Inglés (Cordillera Occidental, Serranía de los Paraguas, a 1 hora en jeep de El Cairo), 2400-2500 m, 30 dic 1986 (fr), Silverstone-Sopkin et al. 2789 (MO). ECUADOR. Bolívar: along first $15 \mathrm{~km}$ of road Chillanes-El Tambo, 01 ${ }^{\circ} 57^{\prime} \mathrm{S}, 7^{\circ} 06^{\prime} \mathrm{W}, 2400 \mathrm{~m}, 18 \mathrm{jul}$ 1991 (fl), van der Werff et al. 12418 (MO). Carchi: Tulcan, Parroquia Chical, sector Gualpi medio, Reserva Indígena Awá, sendero a San Marcos al N de la casa comunal, $01^{\circ} 02^{\prime} \mathrm{N}$, $78^{\circ} 16^{\prime} \mathrm{W}, 1000 \mathrm{~m}, 23-27$ may 1992 (fr), Tipaz et al. 1099 (MO); Cerro Golondrinas, on crest of $\mathrm{N}$ ridge low elfin forest, $0^{\circ} 51^{\prime} 30 " \mathrm{~N}, 78^{\circ} 8^{\prime} 20^{\prime \prime} \mathrm{W}, 2880-2950$ m, 24 jul 1994 (fl), Boyle et al. 3413 (MO). Loja: Cerro Villonaco, new road Loja-La Toma, $\mathrm{km} \mathrm{24.7,} \mathrm{turnoff} \mathrm{toward} \mathrm{Loja} \mathrm{on} \mathrm{old} \mathrm{road,} \mathrm{km} 6$, $4^{\circ} 00^{\prime} 03^{\prime \prime} \mathrm{S}, 79^{\circ} 16^{\prime} 30^{\prime \prime} \mathrm{W}, 2430 \mathrm{~m}, 17$ abr 1994 (fr), Jørgensen et al. 295 (MO); Amaluza, 5-10 km ENE of the village (Pasaje del Romerillo), $4^{\circ} 34^{\prime} \mathrm{S}, 79^{\circ} 23^{\prime} \mathrm{W}, 2400-2700 \mathrm{~m}, 23$ set 1976 (fl), Øllgaard \& Balslev 9742 (MO). COSTA RICA. Alajuela: San Ramón, Estación Biológica Alberto Manuel Brenes, Cuenca del Aranjuez, Arancibia, Fila Cidral, entrando por Corazón de Jesús, $10^{\circ} 14^{\prime} 30^{\prime \prime} \mathrm{N}, 84^{\circ} 42^{\prime} 00^{\prime \prime} \mathrm{W}, 1400 \mathrm{~m}, 11$ oct 1997 (fr), González 2042 (USJ); San Carlos, Peñas Blancas, 1100 m, 10 jul 1985 (fr), Haber \& Bello 1946 (MO); 15 km NNW of San Ramón by road $2.5 \mathrm{~km} \mathrm{~N}$ of Balsa on road to San Lorenzo, $10^{\circ} 11^{\prime} \mathrm{N}, 84^{\circ} 30^{\prime} \mathrm{W}, 1050-1100 \mathrm{~m}, 24$ abr 1983 (fl), Liesner \& Judziewicz 14711 (MO); Upala, Bijagua, El Pilón, Cerro La Carmela entre Río Celeste y cabeceras del Río Chimurria, 1043'15"N, 8459'45"W, 1000 m, 11 jul 1988 (fr), Herrera 2055 (MO); collected along the Rio Zapote about 1 $\mathrm{km}$ from the small town of Zapote $10^{\circ} 45^{\prime} \mathrm{N}, 85^{\circ} 05^{\prime} \mathrm{W}, 500 \mathrm{~m}$, 5 ene 1975 (fr), Taylor 18164 (US). Cartago: Turrialba, San Juan Norte, Montaña de los Ernest, 800 m, 1 ene 1977 (fr), Gómez-Laurito 2229 (USJ); $5.5 \mathrm{~km} \mathrm{~S}$ of Tapantí, $1400 \mathrm{~m}, 8$ oct 1967 (fl), Lent 1394 (MO). Guanacaste: Liberia, Parque Nacional Rincón de la Vieja, Cordillera de Guanacaste, 
Rincón de la Vieja, $10^{\circ} 45^{\prime} 00^{\prime \prime} \mathrm{N}, 85^{\circ} 18^{\prime} 00^{\prime \prime} \mathrm{W}, 600 \mathrm{~m}, 10 \mathrm{mar}$ 1996 (fr), Espinosa 1448 (INB); Liberia, Parque Nacional Guanacaste, Estación Pitilla, Sendero El Mismo, Finca La Pasmompa, $11^{\circ} 02^{\prime} 00^{\prime \prime} \mathrm{N}, 85^{\circ} 24^{\prime} 30^{\prime \prime} \mathrm{W}, 700$ m, 9 dic 1990 (fr), Ríos 215 (CR, INB, MO); Liberia, Parque Nacional Guanacaste, Estación Pitilla, $10^{\circ} 05^{\prime} 02^{\prime \prime} \mathrm{N}, 84^{\circ} 25^{\prime} 40^{\prime \prime} \mathrm{W}, 700 \mathrm{~m}$, 1 abr 1991 (fl), Moraga 382 (USJ); La Chirripa ridge, 4 km NE El Dos de Tilarán, cloud forest exposed to Atlantic trade winds, continental divide, $10^{\circ} 25^{\prime} \mathrm{N}, 84^{\circ} 53^{\prime} \mathrm{W}, 1000 \mathrm{~m}, 8$ may 1986 (fl), Haber et al. 4853 (MO); upper Río Chiquito valley, $5 \mathrm{~km} \mathrm{~N}$ of Santa Elena, Atlantic slope rain forest, $10^{\circ} 25^{\prime} \mathrm{N}$, 84 $50^{\prime} \mathrm{W}, 1300$ m, 13 abr 1986, Haber 4373 (MO). Heredia: Parque Nacional Braulio Carrillo, Estación Magsasay, bosque primario y orillas de potreros, $10^{\circ} 24^{\prime} 03^{\prime \prime} \mathrm{N}, 84^{\circ} 03^{\prime} 03^{\prime \prime} \mathrm{W}, 200$ m, 9 jul 1990 (fl), Alcázar et al. 140 (INB); Sarapiquí, Puerto Viejo, Finca La Selva, the OTS Field Station on the Río Puerto Viejo just E of its junction with the Río Sarapiquí, 100 m, 25 jul 1986 (fl), Wilbur 40453 (CR); Sarapiquí, Chilamate, Finca El Bejuco, 10²7'35"N, 8403'55"W, 75 m, 30 ene 1990 (fr), Chacón 700 (CR, MO). Limón: Reserva Indígena Talamanca Sukut, desembocadura del Río Sukut en el Río Urén, camino al sureste hacia Purisqui, $9^{\circ} 23^{\prime} 30^{\prime \prime} \mathrm{N}$, 82 $58^{\prime} 00^{\prime \prime} \mathrm{W}, 650 \mathrm{~m}, 7$ jul 1989 (fr), Hammel et al. 17592 (MO); Parque Tortuguero, Estación Cuatro Esquinas, primer islote Laguna Tortuguero, frente a la casa-estación, $10^{\circ} 31^{\prime} \mathrm{N}$, $83^{\circ} 30^{\prime} \mathrm{W}, 4$ m, 24 nov 1987 (fr), Robles 1316 (CR, MO); Talamanca, Distrito Bratsi, Reserva Indígena Bribri, $1.7 \mathrm{~km}$ southwest of Kivut, $6.1 \mathrm{~km}$ southwest of Alto Lari, $09^{\circ} 23^{\prime} 35^{\prime \prime} \mathrm{N}, 83^{\circ} 05^{\prime} 50^{\prime \prime} \mathrm{W}, 1400-1500 \mathrm{~m}, 10$ mar 1992 (fr), Schmidt \& Aguilar 644 (CR, INB, MO). Puntarenas: Coto Brus, Parque Internacional La Amistad, Cordillera de Talamanca, Estación Altamira, Sendero Los Gigantes, $09^{\circ} 01^{\prime} 30^{\prime \prime N}, 82^{\circ} 57^{\prime} 40^{\prime \prime}, 1680$ m, 14 abr 1995 (fl), Angulo 177 (INB); Coto Brus, Parque Internacional La Amistad, Cordillera de Talamanca, Las Cruces, $08^{\circ} 47^{\prime} 20^{\prime \prime} \mathrm{N}$, 82 $58^{\prime} 30^{\prime \prime} \mathrm{W}, 1200-1300$ m, 30 jun 1995 (fr), Angulo 393 (INB); Golfito, Península de Osa, Parque Nacional Corcovado, Sendero Sirena, $8^{\circ} 28^{\prime} 48^{\prime \prime} \mathrm{N}, 83^{\circ} 35^{\prime} 28^{\prime \prime} \mathrm{W}, 1-100$ m, 25 mar 1997 (fr), Aguilar 5012 (INB); Fila El Tigre, SE of Las Alturas, $c a .8^{\circ} 56^{\prime} \mathrm{N}, 82^{\circ} 51^{\prime} \mathrm{W}, 1350-1450 \mathrm{~m}, 29$ ago 1983 (fr), Davidse 24212 (CR, MO); Puntarenas, Reserva Biológica Monteverde, Cordillera de Tilarán, Estación Aleman's, $10^{\circ} 17^{\prime} 40^{\prime \prime} \mathrm{N}, 84^{\circ} 45^{\prime} 00^{\prime \prime} \mathrm{W}, 1000 \mathrm{~m}, 10$ set (fr), Bello et al. 4045 (INB); Punta Banco, 0-200 m, 22 ago 1988 (fr), Chavarría et al. 282 (CR, MO); Monteverde Cloud Forest Reserve, Pacific slope, lower montane wet forest, $10^{\circ} 20^{\prime} \mathrm{N}$, 84 $4^{\circ} 50^{\prime} \mathrm{W}, 1500$ m, 12 ene 1986 (fl), Haber 4250 (MO). San José: Aserrí, Zona Protectora Cerros de Escazú, Cerros Escazú, La Carpintera, Cerro Daser, Alto Hierbabuena, $9^{\circ} 50^{\prime} 30^{\prime \prime} \mathrm{N}, 8^{\circ} 07^{\prime} 20^{\prime \prime} \mathrm{W}, 2200 \mathrm{~m}, 1$ abr 1995 (fl), Morales \& Bohs 3852 (CR, INB, MO); El Pizote, San Ramón de Tres Ríos, 1500 m, 26 jun 1984 (fr), Herrera \& Gómez 22861 (CR, MO, USJ); Vasquez de Coronado Cantón, Braulio Carrillo National Park, along San José to Siquerres Hwy., along trail to Río Sucio, site of the Old Carrillo Station, $10^{\circ} 09^{\prime} 50 " \mathrm{~N}$, 8357'10"W, 600-700 m, 30 ago 1996 (fr), Croat 78782 (MO); Cerros de La Palma, 1300 m, dic 1913 (fl), Jiménez 938 (US). EL SALVADOR. Santa Ana: Hacienda Montecristo n.
Metapán, 2200 m, 27 ago 1951, Rohweder 989 (MO). GUATEMALA. Baja Verapaz: Purulhá, along highway CA 14 between El Progreso and Cobán, 3 mi S Purulhá, 17 mi N of junction with Hwy, 17 to Salamá and San Jerónimo vic. km marker 160 , W of hwy, $15^{\circ} 13^{\prime} \mathrm{N}, 90^{\circ} 12^{\prime} \mathrm{W}, 1620-1720 \mathrm{~m}, 26$ ene 1987 (fr), Croat et al. 63767 (MO). Izabal: Puerto Barrios, en el Río Las Escobas, camino entre Puerto Barrios y Punta de Palma, 120 m, 10 set 1988 (fl), Martínez et al. 23657 (MO). Petén: Camino para Puerto Méndez, km 129, lado sureste del camino, 8 ene 1972 (fr), Ortíz 2182 (MO); Dolores, about 3 $\mathrm{km}$ se.e. in pineland, 19 ago 1961 (fl), Contreras 2722 (MO). HONDURAS. Comayagua: In thicket near El Rincón, 10 miles W of Siguatepeque, 1400-1500 m, 24 jul 1936 (fr), Yuncker et al. 6051 (MO). Francisco Morazán: Cloud forest area in mountains above San Juancito, 2000 m, 25 mar 1948 (fl), 11775 (MO). Olancho: Montaña de Chifiringo, bosque de hoja ancha, $20 \mathrm{~km} \mathrm{~S}$ de Campamento, 1079 m, 6 oct 1979 (fr), Soto 127 (MO). MÉXICO. Chiapas: Acagoyagua, Escuintla, 12 ago 1947 (bo, fl), Matuda 16826 (USJ); Trinitaria, slopes with montane rain forest Liquidambar, Magnolia, Vochysia, east of Laguna Tzikaw, Monte Bello National Park, 1300 m, 23 ene 1973 (fr), Breedlove 32258 (MO). Veracruz: San Andrés Tuxtla, $2 \mathrm{~km}$ al $\mathrm{N}$ de la Estación de Biología Tropical Los Tuxtlas, $18^{\circ} 34^{\prime} \mathrm{N}$ y $18^{\circ} 36^{\prime} \mathrm{N}, 95^{\circ} 04^{\prime} \mathrm{W}$ y $95^{\circ} 09^{\prime} \mathrm{W}, 25$ set 1986 (fl), Cedillo 3682 (MO); forest near Jalapa, $4000 \mathrm{ft}, 21$ may 1899 (fl), Pringle 7780 (MO). NICARAGUA. Boaco: Hacienda Rancho Grande, $c a .16 \mathrm{~km}$ al N de Comoapa, $12^{\circ} 33^{\prime} \mathrm{N}, 85^{\circ} 30^{\prime} \mathrm{W}, 27$ dic 1984 (fr), Estrada et al. 99 (MO). Jinotega: N slope of Volcán Yalí, ca. $13^{\circ} 15^{\prime} \mathrm{N}, 86^{\circ} 10^{\prime} \mathrm{W}$, 1200-1400 m, 25 oct 1979 (fr), Stevens et al. 15107 (MO). Matagalpa: Camino al cerro Carlota a $2 \mathrm{~km}$ de la carretera al Tuma, $12^{\circ} 58^{\prime} \mathrm{N}, 85^{\circ} 52^{\prime} \mathrm{W}, 1040-1100 \mathrm{~m}, 5$ mar 1982 (fr), Moreno 15632 (MO). PANAMÁ. Bocas del Toro: N coast of Escudo de Veraguas Island, $9^{\circ} 05^{\prime} \mathrm{N}, 81^{\circ} 35^{\prime} 2 \mathrm{~W}, 5 \mathrm{~m}, 8$ ago 1987 (fl), McPherson 11434 (MO). Chiriquí: Vicinity of Fortuna Dam, along trail across valley $\mathrm{S}$ of lake, forest, $8^{\circ} 45^{\prime} 04^{\prime \prime N}, 82^{\circ} 15^{\prime} 04^{\prime \prime W}, 1300-1400 \mathrm{~m}, 7$ feb 1987 (fr), McPherson 10392 (MO).

2. Smilax mollis Humb. \& Bonpl. ex Willd., Sp. Pl. 4: 785. 1806. TIPO: MÉXICO. Veracruz: Jalapa, Humboldt \& Bonpland s. $n$. (holotipo, P, [IDC microficha 1440.1338.II.9).

FIG. 4

Smilax angustiflora A. DC., Monogr. Phan.1: 67. 1878. TIPO: Costa RicA. Alto de la Cruz, en Azarí, Hoffmann 575 (holotipo, B; isotipos [fragmentos G, US!], [foto 10052 F!]); syn. nov.

Smilax candelariae A. DC., Monogr. Phan. 1: 70.

1878. TIPO: Costa Rica. Candelaria, planta femenina, Hoffmann s. $n$. (holotipo, B [foto 10054 F!]); syn. nov.

Smilax mollis var. acuminata A. DC., Monogr. Phan. 1: 68. 1878. TIPO: México. Veracruz, Orizaba, Bourgeau 3038 (K, P [fragmento US, 
imagen digital US!]).

Smilax ovata Sessé \& Moc., Fl. Mexic. (ed. 2) 232.

1894. TIPO: México, Sessé \& Mociño s. n. (G).

Smilax gymnopoda Apt, Repert. Spec. Nov. Regni

Veg. 18: 401. 1922. TIPO: México. Veracruz, in

Dickichten nahe Jalapa in einer Höhe von 1200

$\mathrm{m}$, Pringle 8130 (lectotipo designado aquí, GH!; isolectotipos, MO!, BM, BR, F, G, GH!, K, NY, $\mathrm{P}$ - imagen digital US!, W).

Smilax gentlei Lundell, Wrightia 3(8): 163. 1966.

TIPO: Belize. Toledo Distr., between Orange

Point and Rio Moho in broken cohune ridge, 12

Abr 1952, P.H. Gentle 7648 [holotipo LL, isotipos LL - foto MO!, S (2 hojas)].

Smilax mollis var. hirsutior Killip \& C. Morton,

Publ. Carnegie Inst. Wash. 461: 288. 1936.

Smilax hirsutior (Killip \& C. Morton) C. Morton.

TIPO: Costa Rica. Cartago, Río Turrialba, Mar

1894, J.D. Smith 4971 (holotipo US!); syn. nov.

Rizoma con engrosamientos en los nudos, sin escamas, raíces cilíndricas, cubriendo casi todo el rizoma. Tallos cilíndricos, finamente pubescentes, a veces glabrescentes, inermes, ocasionalmente morados cuando están jóvenes. Hojas ovadas a lanceoladas, 7 19 x 3.4-11 cm, 7 nervios, raramente trinervada, los nervios primarios prominentes en el envés, conectados por nervaduras reticuladas, conspicuas, ápice agudo, base cordada, margen entero, muy pubescentes las jóvenes, finamente tomentosas las adultas, cartáceas a subcoriáceas, algo rugosas; pecíolo 0.8-3 $\mathrm{cm}$ de largo, pubescente. Umbelas solitarias, rara vez saliendo tres de la base de la rama floral; las estaminadas con pedúnculo cilíndrico, $0.5-2.5 \mathrm{~cm}$, más largo o más corto que el pecíolo subyacente; tépalos 4-7 mm, filamentos 2-4 mm, anteras 1-1.5 mm, más cortas que los filamentos; las pistiladas con pedúnculo aplanado, 0.3-3 cm, más largo o corto que el pecíolo subyacente; tépalos 3-3.5 mm. Bayas $0.5-1 \mathrm{~cm}$ de diámetro, anaranjadas.

DISTRIBUCIÓN Y HÁBITAT. Se encuentra en bosques húmedos, montanos, premontanos, entre 0 y $2000 \mathrm{~m}$ (Fig. 3). Se distribuye desde México hasta Panamá (Huft 1994).

Smilax mollis se caracteriza por tallos cilíndricos, ausencia de aguijones y hojas jóvenes muy pubes- centes. En lugares abiertos sus hojas suelen ser grandes, rugosas y casi glabras o finamente pubescentes, con agallas cilíndricas pubescentes, pedúnculos iguales o más largos que el pecíolo subyacente, tépalos 3-7 $\mathrm{mm}$ y frutos anaranjados.

Esta especie presente una alta variabilidad morfológica, tanto vegetativa como reproductiva. A continuación se presentan varias observaciones:

1. Los nombres que se consideran sinónimos presentan ejemplares tipo que se diferencian por la cantidad de pubescencia y el tamaño de las hojas. De Candolle (1878) señala que Smilax angustiflora se encontraba mezclada en el herbario con $S$. tomentosa; que se diferencia de ésta por las hojas pálidas ovadas, con base laminar cordada, nervios medios profundamente connados, consistencia menos firme, pedúnculos más largos, botones reducidos y anteras mutiladas. Smilax candelariae también había sido confundida en herbario con $S$. tomentosa Kunth y $S$. angustiflora.

De Candolle (1878) enfatiza que $S$. candelariae es diferente de $S$. tomentosa y $S$. angustiflora por las semillas, aunque sólo hace mención de los frutos negruzcos cuando están secos y los pedúnculos más cortos que los pecíolos subyacentes. Además, sugiere que tal vez el espécimen con frutos de Jurgensen 563 (FI-W), recolectado en México, podría ser también esta especie. Huft (1994) indica que es endémica en Costa Rica y completa la descripción con las bayas de color rojo.

2. Al examinar ejemplares de $S$. mollis se observa que en la parte superior del tallo de especímenes de herbario se encuentran pedúnculos muy cortos en comparación con los pecíolos, pero en las ramas viejas los pedúnculos son más largos que los pecíolos; por tanto, esto forma parte de una variación morfológica. La falta de observaciones en el campo y la poca disponibilidad de especímenes recolectados en años anteriores ha hecho que algunas especies no hayan sido apropiadamente descritas. Ejemplo de ello son S. mollis var. acuminata y S. hirsutior.

3. La dioecia de Smilax ha representado un problema en las descripciones. Smilax hirsutior y S. angustiflora están descritas sólo con base en flores masculinas y $S$. candelariae con base en flores femeninas. El tipo de la última está representado sólo por 

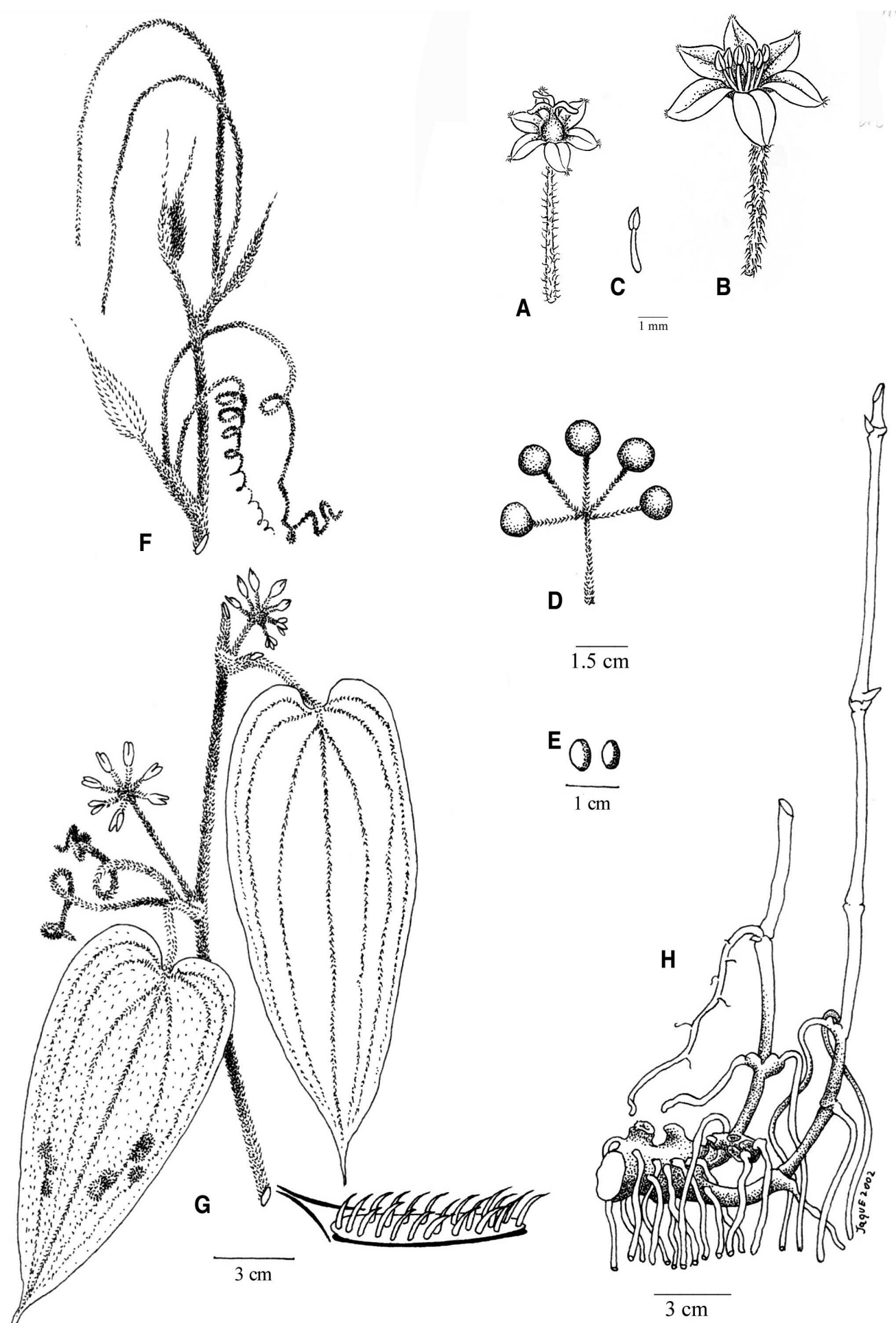

Figura 4. Smilax mollis Humb. \& Bonpl.: A. Flor femenina, B. Flor masculina, C. Estambre, D. Frutos, E. Semillas, F. Rama joven, G. Rama fértil con agallas y botones, H. Tallo y rizoma 
fragmentos de la planta. Ambas situaciones han representado un problema en la descripción de especies nuevas, porque sus caracteres se han basado en un solo sexo de la planta.

4. El color de los frutos se ha usado para distinguir las especies pubescentes de Smilax. Esta coloración del fruto quizás depende de la observación del recolector, del ambiente donde se desarrolla el bejuco y de la variación del color de las bayas a lo largo del desarrollo.

ESPECÍMENES EXAMINADOS. BELICE. Cayo: Chaa Creek, $17^{\circ} 06^{\prime} \mathrm{N}, 89^{\circ} 04^{\prime} \mathrm{W}, 8$ dic 1992 (fr), Arvigo \& Cocon 694 (MO). COSTA RICA. San Ramón, Reserva Biológica Alberto Manuel Brenes, Cordillera de Tilarán, camino de entrada a la estación, $10^{\circ} 13^{\prime} 00^{\prime \prime} \mathrm{N}, 84^{\circ} 37^{\prime} 00^{\prime \prime} \mathrm{W}, 850 \mathrm{~m}, 6$ jun 1998 (fr), Gómez-Laurito et al. 13120 (USJ); $31 / 2 \mathrm{~km}$ west of Fortuna, $2 \mathrm{~km}$ nortwest of New Volcán Arenal along its sloping base, $10^{\circ} 28^{\prime} \mathrm{N}, 84^{\circ} 41^{\prime} \mathrm{W}, 1500 \mathrm{~m}, 5$ ago 1972 (fr), Taylor \& Taylor 11678 (MO); along road from San Ramón northward through Balsa, ca. $5.7 \mathrm{~km} \mathrm{~N}$ of bridge over Quebrada Volio, SW of road, $10^{\circ} 08^{\prime} \mathrm{N}, 84^{\circ} 29^{\prime} \mathrm{W}, 1100-1150 \mathrm{~m}, 10$ set 1979 (fr), Stevens 14176 (MO). Cartago: Cartago, Pacayas, 1170 m, 29 jun 1952 (fr), Córdoba 168 (USJ); Turrialba, Instituto Interamericano de Ciencias Agrícolas, 600 m, 28 set 1950, León 2778 (USJ); Forest on Casa de Tejas Ridge, above Río Gato, $9^{\circ} 47^{\prime} \mathrm{N}, 83^{\circ} 41^{\prime} \mathrm{W}, 800 \mathrm{~m}, 15$ set 1973 (fr), Lent 3606 (CR). Heredia: Sarapíquí, Puerto Viejo, Finca La Selva, the OTS Field Station on the Río Puerto Viejo just E of its junction with the Río Sarapiquí, 100 m, 4 jun 1985 (fr), Jacobs et al. 3241 (USJ); Parque Nacional Braulio Carrillo, Estación El Ceibo, sendero $100 \mathrm{~m}$ después del puesto, en potrero de caba$1 \mathrm{los}, 10^{\circ} 19^{\prime} 40^{\prime \prime} \mathrm{N}, 84^{\circ} 04^{\prime} 50^{\prime \prime} \mathrm{W}, 500 \mathrm{~m}, 7$ nov $1992(\mathrm{fl})$ Fernández 461 (INB, MO). Guanacaste: Liberia, Parque Nacional de Guanacaste, Estación Cacao, bosque primario, potreros y orilla de bosque, $10^{\circ} 55^{\prime} 45^{\prime \prime} \mathrm{N}, 85^{\circ} 28^{\prime} 15^{\prime \prime} \mathrm{W}, 1100 \mathrm{~m}$, 3 nov 1990 (fr), Alcázar 31 (CR); Parque Nacional Guanacaste, La Cruz, Estación Pitilla $10^{\circ} 59^{\prime} 26^{\prime \prime} \mathrm{N}$, $85^{\circ} 25^{\prime} 40^{\prime \prime} \mathrm{W}, 700 \mathrm{~m}, 7$ nov 1990 (fr), Moraga 152 (INB); Tilarán, Cuenca del San Carlos, sector Lago Cote, en bosque primario y secundario, $10^{\circ} 34^{\prime} 15^{\prime \prime} \mathrm{N}, 84^{\circ} 54^{\prime} 50^{\prime \prime} \mathrm{W}, 680 \mathrm{~m}, 12$ mar 2000 (fr), Chávez 305 (INB); P. N. Rincón de La Vieja, Sector el Canal, a $1 \mathrm{~km}$ aguas arriba de la administración, $10^{\circ} 46^{\prime} 09^{\prime \prime} \mathrm{N}, 85^{\circ} 17^{\prime} 25^{\prime \prime} \mathrm{W}, 900 \mathrm{~m}, 2$ abr 1991 (fr), Rivera 1214 (INB, MO). Limón: Borde de Lago Dabagri, 1100 m, 4 nov 1984 (bo, fl), Gómez et al. 23139 (CR); Limón, Cuenca del Estrella, Penhurst, Finca Júpiter, $9^{\circ} 43^{\prime} 52^{\prime \prime N}, 82^{\circ} 55^{\prime} 56^{\prime \prime} \mathrm{W}, 200$ m, 18 ene 1997 (fr), Rodríguez 1912 (CR, INB, MO); Talamanca, Reserva Indígena Kekoldi, en el camino hacia la casa de doña Ana, 9³7'N, 82²3'W, 150 m, 10 jun 1996 (fl), Dalle \& Barrantes 102 (USJ); Cerro Coronel, E of Río Zapote, E of new road, higher parts of hill ca $1.5 \mathrm{~km}$ from Río Colorado, $10^{\circ} 40^{\prime} \mathrm{N}, 83^{\circ} 40^{\prime} \mathrm{W}, 40-100 \mathrm{~m}, 13$ mar 1987 (fr), Stevens et al. 24757 (MO). Puntarenas: Coto Brus, San Vito de Java, Estación Biológica Las Cruces, $20 \mathrm{~m}$ uphill from Trail Marker ft $12,8^{\circ} 47^{\prime} 8.9^{\prime \prime} \mathrm{N}, 82^{\circ} 57^{\prime} 29.3^{\prime \prime} \mathrm{W}, 1030 \mathrm{~m}, 8 \mathrm{mar}$
1996 (fr), Krings 254 (USJ); Garabito, Reserva Biológica Carara, Valle del Tárcoles, Bijagual, alrededores de Quebrada Máquina, bosque secundario, 9 9 $45^{\prime} 00^{\prime \prime} \mathrm{N}, 84^{\circ} 33^{\prime} 50^{\prime \prime} \mathrm{W}, 500 \mathrm{~m}$, 2 jul 1990 (fr), Rojas 98 (INB); Monteverde, along road from the Tropical Science Center to Monteverde tropical cloud forest, $10^{\circ} 18^{\prime} 10^{\prime \prime} \mathrm{N}, 84^{\circ} 47^{\prime} 30^{\prime \prime} \mathrm{W}, 1400 \mathrm{~m}, 14$ jul 2000 (fr), Boyle 5732 (USJ); Osa, Reserva Forestal Golfo Dulce, Cuenca Térraba-Sierpe, Bahía Chal, entrada a Chocuaco, $8^{\circ} 43^{\prime} 50 " \mathrm{~N}$, 83 $3^{\circ} 27^{\prime} 17^{\prime \prime W}, 150-175$ m, 25 jun 1998 (fr), Aguilar 5453 (INB); Puntarenas, Monteverde, community near Quaker school, $10^{\circ} 18^{\prime} \mathrm{N}, 84^{\circ} 48^{\prime} \mathrm{W}, 1400-1500 \mathrm{~m}, 3$ jul 1991 (fl), Haber \& Zuchowski 10713 (CR, INB, MO); road 2-4 km N of Santa Elena, forested roadsides and remnant forest stands, 1500-1600 m, 4 may 1984 (fr), Murphy \& Whetstone 833 (MO); Parque Nacional Corcovado, Sirena, look out trail, $8^{\circ} 28^{\prime} \mathrm{N}, 83^{\circ} 55^{\prime} \mathrm{W}, 1-20 \mathrm{~m}, 21$ oct 1989 (fr), Kernan et al. 1300 (CR, MO); Buenos Aires, Cuenca Térraba-Sierpe, Puesto Tres

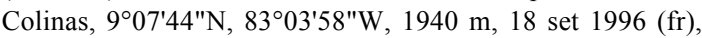
Gamboa \& Picado 671 (INB, MO). San José: Aserrí, $3 \mathrm{~km}$ al $\mathrm{S}$ de Aserrí, 1700 m, 8 feb 1977 (fr), Gómez-Laurito \& Valerio 2372 (USJ); Nordabfall der Cerros de Escazú, Alto Tapezco, südlich oberhalb Bebedero (oberhalb Escazú), 16001740 m, 21 nov 1992 (fl), Döbbeler 5463 (CR); Pérez Zeledón, Río Nuevo, Reserva Forestal Los Santos, camino entre California y Zaragoza, $9^{\circ} 28^{\prime} 10^{\prime \prime} \mathrm{N}, 8^{\circ} 49^{\prime} 20^{\prime \prime} \mathrm{W}, 1000 \mathrm{~m}$, 6 mar 2001 (fr), Quesada et al. 477 (CR); San Marcos de Tarrazú, Estribación fila San Isidro, bosques residuales en zona muy alterada, $9^{\circ} 33^{\prime} 50^{\prime \prime} \mathrm{N}, 84^{\circ} 04^{\prime} 40^{\prime \prime} \mathrm{W}, 1000-1100 \mathrm{~m}, 29$ nov 1995 (fr), Cascante et al. 889 (CR); Zona Protectora La Cangreja, Mastatal de Puriscal, bosque primario en las márgenes del Río Negro, $9^{\circ} 40^{\prime} 55^{\prime \prime} \mathrm{N}, 8^{\circ} 23^{\prime} 38^{\prime \prime} \mathrm{W}, 290 \mathrm{~m}, 8$ nov 1992 (fr), Morales 983 (INB, MO); Reserva Biológica Carara, sector Bijagual, sitio Quebrada Máquina, $9^{\circ} 45^{\prime} 00^{\prime \prime} \mathrm{N}$, 84³3'50"W, 450 m, 2 nov 1990 (fr), Zúniga \& Varela 334 (CR, MO); Reserva Biológica Carara, Estación Bijagual, $9^{\circ} 46^{\prime} \mathrm{N}, 84^{\circ} 36^{\prime} \mathrm{W}, 500 \mathrm{~m}, 23$ jul 1990 (fr), Bello 2339 (INB, MO). GUATEMALA. Izabal: El Estro La Mina de Exmibal, al E del Estor, $15^{\circ} 31^{\prime} \mathrm{N}, 8^{\circ} 23^{\prime} \mathrm{W}, 50 \mathrm{~m}, 17$ jul 1988 (fr), Tenorio et al. 14576 (MO); Puerto Barrios, a $3 \mathrm{~km}$ al S de Punta de Palma, 5 m, 9 set 1988 (fr), Martínez et al. 23627 (MO). HONDURAS. Atlántida: La Ceiba, on the mountain slopes and coastal plains, jun a ago 1938 (bo), Yuncker et al. 8271 (MO). Colón: $1.8 \mathrm{mi}$ strip on the bank of Río Guaimoreto between old brigde and Laguna Guaimoreto 4.5 $\mathrm{mi}, \mathrm{NE}$ of Trujillo on old road to Castilla, $15^{\circ} 57^{\prime} 30^{\prime \prime} \mathrm{N}$, $85^{\circ} 54^{\prime} 30^{\prime \prime W}, 20$ nov 1980 (fr), Saunders 715 (MO). MEXICO. Chiapas: Ocosingo, 6-8 $\mathrm{km} \mathrm{N}$ of Ocosingo along road to bachajón, 900 m, 24 set 1972 (fr), Breedlove 27955 (MO); along highway 195 between Chiapa de Corzo and Pichucalco, $7.9 \mathrm{mi} \mathrm{N}$ of Bochil, $17^{\circ} 03^{\prime} \mathrm{N}, 92^{\circ} 51^{\prime} \mathrm{W}, 1990 \mathrm{~m}, 17 \mathrm{feb} 1987$ (fr), Croat \& Hannon 65086 (MO). Veracruz: San Andrés Tuxtla, Estación de Biología Tropical Los Tuxtla, 18³4' y $18^{\circ} 36^{\prime} \mathrm{N}, 95^{\circ} 04^{\prime}$ y $95^{\circ} 09^{\prime} \mathrm{W}, 160 \mathrm{~m}, 15$ feb 1985 (fr), Ibarra Manríquez \& Sinaca Colín 2281 (MO). Oaxaca: María Chimalapa, Arroyo Palomares, ca. $2 \mathrm{~km}$ al N de Santa María, $16^{\circ} 55^{\prime} \mathrm{N}, 94^{\circ} 41^{\prime} \mathrm{W}, 250 \mathrm{~m}, 8$ dic $1984(\mathrm{fl})$, Hernández 630 (CHAPA, MO). NICARAGUA. Río San Juan: Between Río Santa Cruz and Caño Santa Crucita, La Palma, $11^{\circ} 2-4^{\prime} \mathrm{N}$, 
84²4-26'W, 40-60 m, 30 nov-2 dic 1984 (fr), Stevens 23447 (MO). Zelaya: Sector Mina Nueva América, $13^{\circ} 45^{\prime} \mathrm{N}$ $84^{\circ} 30^{\prime} \mathrm{W}, 500$ m, 22 set 1984 (fr), Ortíz 2135 (MO); Cerro Saslaya, $20 \mathrm{~km} \mathrm{~W}$ of Siuna, 1100-1400 m, 5 may 1977 (fl), Neill 1834 (MO). PANAMA. Chiriquí: Vicinity of Fortuna Dam, along trail from higway across Rio Hornito, $\mathrm{S}$ of reservoir, $8^{\circ} 45^{\prime} \mathrm{N}, 82^{\circ} 15^{\prime} \mathrm{W}, 1100-1350 \mathrm{~m}, 7$ set 1987 (fl), McPherson 11670 (MO). Colón: rain forest along Santa Rita Ridge, road to Estacion Calibrar Lluvia el Agua Clara $9^{\circ} 22^{\prime} \mathrm{N}$, 7942-45'W, 1300 m, 26 jun 1971 (fl, fr), Webster \& Dressler 16737 (MO) Panamá: Road from Panamerican Highway to Cerro Jefe, summit, $23.4 \mathrm{~km}$ from Panamerican Highway, 22 jun 1977 (fr), Folsom 3829 (MO); Barro Colorado Island, Gatun Lake, 8-10 ago 1940 (fl, fr), Barlett \& Lasser 16721 (MO).

3. Smilax panamensis Morong, Bull. Torrey Bot. Club 21: 441. 1894. TIPO: PANAMÁ. Gatún Station, Hayes 63 (Lectotipo, designado por Killip \& C. Morton, Publ. Carnegie Inst. Wash. 461: 275 (1936), imagen digital $\mathrm{NY}$ !).

FIG. 5

Smilax ramonensis Apt, Repert. Spec. Nov. Regni Veg. 18: 405. 1922. TIPO: Costa Rica: San Pedro, near San Ramón, 1400-1600 m, Tonduz 17723 (holotipo B; isotipos, BM, BR, CR!, fragmentos y foto digital, US).

Rizoma con engrosamiento tuberoso, blanco, escamas café oscuro, raíces cilíndricas, a veces con escamas. Tallos cilíndricos, glabros, armados en la parte inferior con aguijones rectos o recurvados, robustos, inermes en la parte superior. Hojas ovadas a lanceoladas, 8-18 x 2-9 cm, 5 nervios desde la base, 2 nervios submarginales conectados por nervaduras reticuladas, conspicuas, ápice acuminado a brevicuspidado, base aguda, margen entero; pecíolos 1-2.5 $\mathrm{cm}$. Umbelas racemosas o solitarias con brácteas prominentes, persistentes en la madurez; las estaminadas con pedúnculo aplanado, $1-3 \mathrm{~cm}$, más largo que el pecíolo subyacente; tépalos de las flores masculinas 3-4 mm, filamentos $c a .1 \mathrm{~mm}$, anteras $c a .2 \mathrm{~mm}$, más largas que los filamentos; las pistiladas con pedúnculo aplanado, 1-2 cm, más largo que el pecíolo subyacente; tépalos 4-5 mm. Bayas 0.7-0.9 mm de diámetro, anaranjadas.

DISTRIBUCIÓN Y HÁBITAT. Es un bejuco abundante en bosques húmedos, premontanos, de 0 a $1700 \mathrm{~m}$. Se encuentra en el noroeste, centro y sur del país (Fig. 7). Se distribuye desde Honduras hasta Panamá.

NOMBRES COMUNES. Cuculmeca, cuculmeca blanca.
Smilax panamensis se caracteriza por el rizoma tuberoso, blanco, tallos redondos con aguijones grandes y rectos, hojas coriáceas, las hojas viejas se tornan amarillas con manchas café, en las muestras de herbario las hojas a veces suelen tornarse negras; agallas como ampollas, de color café pálido prominentes en el haz de la hoja; el pedúnculo es más largo que el pecíolo subyacente; umbelas en racimo con brácteas persistentes en la inflorescencia, tépalos $c a$. $4 \mathrm{~mm}$ y bayas anaranjadas.

Morong (1894) describe esta especie con tallos inermes, ligeramente pubescentes, pedúnculos y zarcillos casi glabros y frutos negros con rojo matizado. Killip \& Morton (1936) sugieren que existe una confusión en los datos originales de la publicación de esta especie, porque el ejemplar recolectado por Hayes 209, con flores pistiladas y frutos, es un espécimen de $S$. mollis y es diferente de los dos especímenes citados (Hayes 63, 68). Estos tres especímenes fueron reexaminados por J.B. Norton en el Jardín Botánico de New York.

Killip \& Morton (1936) aclaran que algunos especímenes de $S$. spissa se encontraban identificados como $S$. panamensis; aunque estas especies crecen asociadas, ambas difieren en algunas características importantes.

ESPECÍMENES EXAMINADOS. COSTA RICA. Alajuela: San Ramón, Reserva Biológica Alberto Manuel Brenes, Cordillera de Tilarán, camino de entrada a la estación, $10^{\circ} 13^{\prime} 00^{\prime \prime} \mathrm{N}, 84^{\circ} 37^{\prime} 00^{\prime \prime} \mathrm{W}, 800-850 \mathrm{~m}, 27$ ago 1995 (fr), Gómez-Laurito \& Mora 12811 (USJ); San Ramón, 22 may 1913, Tonduz 35417 (MO); San Ramón, Piedades Norte, 1100 m, 1 feb 1984, Khan et al. 722 (CR, MO, USJ). Cartago: Monumento Nacional Guayabo, Cañón del Río Guayabo, 550 m, 1 feb 1990 (fr), Sánchez 62 (USJ). Guanacaste: 2 km al NE de Tilarán, 880 m, 4 dic 1963 (fr), Jiménez 1343 (CR); 15 km NNW of San Ramón by road, $2.5 \mathrm{~km} \mathrm{~N}$ of Balsa on road to San Lorenzo, $10^{\circ} 22^{\prime} \mathrm{N} 84^{\circ} 30^{\prime} \mathrm{W}, 1050-1100 \mathrm{~m}, 25$ abr 1983 (fr), Liesner \& Judziewicz 14889 (MO); San Ramón, Cerro de Santiago, 1100 m, 29 abr 1982 (fr), Carvajal 217 (CR, MO, USJ); Parque Nacional Guanacaste, Estación Pitilla, Sendero Laguna, $10^{\circ} 59^{\prime} 26^{\prime \prime} \mathrm{N}, 85^{\circ} 25^{\prime} 40^{\prime \prime} \mathrm{W}, 700-1000 \mathrm{~m}, 2$ ene 1991 (fr), Moraga 268 (INB, MO). Heredia: Sarapiquí, Puerto Viejo, Estación Biológica La Selva, 25 jun 1983 (fr), Chacón 992 (CR). Limón: Cordillera de Talamanca, along ridge between Quebrada Camagre and Río Barbilla, $10^{\circ} 00^{\prime} \mathrm{N}$, $83^{\circ} 24.5^{\prime} \mathrm{W}, 180-480 \mathrm{~m}, 9$ set 1998 (fr), Grayum et al. 8902 (INB); Parque Nacional Tortuguero, Estación Agua Fría, Sendero Agua Fría, hasta entrada Sendero Aguacate, $10^{\circ} 26^{\prime} 40^{\prime \prime} \mathrm{N}, 83^{\circ} 34^{\prime} 40^{\prime \prime} \mathrm{W}, 20 \mathrm{~m}, 1$ dic 1990 (fr), Solano 245 


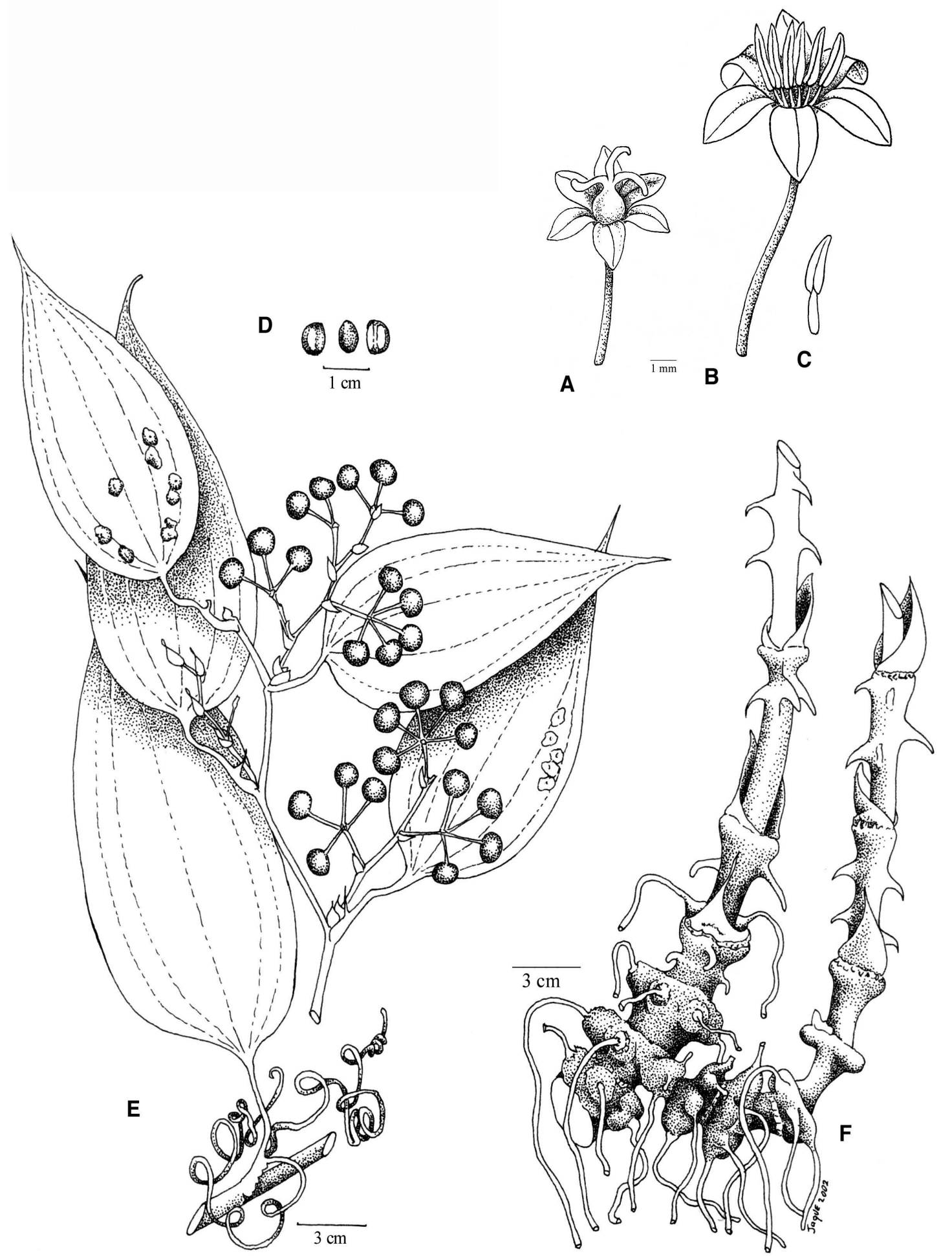

Figura 5. Smilax panamensis Morong: A. Flor femenina, B. Flor masculina, C. Estambre, D. Semillas, E. Rama fértil con agallas y frutos, F. Tallo y rizoma. 
(INB). Puntarenas: Buenos Aires, Parque Internacional La Amistad, Cordillera de Talamanca, Estación Altamira, Sendero Los Gigantes, bosque secundario, $9^{\circ} 01^{\prime} 35^{\prime \prime} \mathrm{N}$, $83^{\circ} 00^{\prime} 45^{\prime \prime} \mathrm{W}, 1250-1300 \mathrm{~m}, 8$ abr 1996, Villalobos 287 (CR, INB, MO); Coto Brus, Parque Internacional La Amistad, Cordillera de Talamanca, Estación Pittier, Aguas Calientes, Rivera Río Cotón y alrededores Aguas Termales, $9^{\circ} 01^{\prime} 30^{\prime \prime} \mathrm{N}$, $82^{\circ} 57^{\prime} 40 " \mathrm{~W}, 1680 \mathrm{~m}, 1 \mathrm{feb} 1995$, Madrigal et al. 23 (INB); Coto Brus, Las Alturas Biological Station, $50 \mathrm{~m}$ along path A, hanging directly above path, premontane wet forest, Canopy entirely open directly above plant, $8^{\circ} 57^{\prime} 12^{\prime \prime} \mathrm{N}, 82^{\circ} 50^{\prime} 10^{\prime \prime} \mathrm{W}$, 1550 m, 10 jul 1998 (fr), Boyle et al. 5627 (USJ); Golfito, Parque Nacional Corcovado, Península de Osa, Estación Sirena, Sendero Naranjos, $8^{\circ} 28^{\prime} 50^{\prime \prime} \mathrm{N}, 8^{\circ} 35^{\prime} 30^{\prime \prime} \mathrm{W}, 10 \mathrm{~m}, 5$ feb 1994 (bo, fl), Aguilar 3075 (CR, INB, MO); Golfito, Mata Palo, Finca Jilva. Jiménez, $8^{\circ} 23^{\prime} 35^{\prime \prime} \mathrm{N}, 83^{\circ} 18^{\prime} 10^{\prime \prime} \mathrm{W}, 200-220$ m, 11 set 1991 (fr), Aguilar 403 (CR, INB, MO); Forest to the E of hairpin ca. $1 \mathrm{~km} \mathrm{SE}$ of Las Alturas de Coto Brus, on road from San Vito, $8^{\circ} 56^{\prime} \mathrm{N}, 82^{\circ} 50^{\prime} \mathrm{W}, 1390-1440 \mathrm{~m}, 13$ jul 1985 (fr), Grayum \& Hammel 5669 (MO). San José: Vicinity of El General, 825 m, feb 1936 (fl); Skutch 2583 (MO); Santa Rosa de Puriscal, Río Negro, faldas Cerro La Cangreja, 400-700 m, 20 jun 1986, Chacón \& Chacón 1949 (CR, MO, USJ). HondurAs. Atlántida: Thicket near Lancetilla, 90 pies, 27 jul 1934 (fr), Yuncker 4776 (MO). Nicaragua. Zelaya: Río Punta Gorda, Atlanta, "La Richard" $200 \mathrm{~m}$, al SE, $11^{\circ} 32^{\prime} \mathrm{N}$, $84^{\circ} 05^{\prime} \mathrm{W}, 20 \mathrm{~m}, 13$ nov 1981 (fr), Moreno \& Sandino 13022 (MO); E del poblado de Nueva Atlanta, $11^{\circ} 34^{\prime} \mathrm{N}, 84^{\circ} 26^{\prime} \mathrm{W}, 20$ feb 1994 (bo, fl), Rueda et al. 3272 (MO); camino a la largo del Río Punta Gorda entre la Corriente la Guitarrona y San

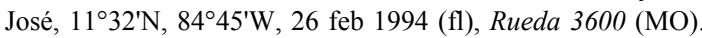
PanAmá. Coclé: $7 \mathrm{~km}$ N of Llano Grande on road to Coclesito, in forest, $1700 \mathrm{ft}$., 8 mar 1978 (fl), Hammel 1933 (MO). Darién: Vicinity of El Real, Río Tuira, S of El Real, Don Pablo Othon's pasture, footthills of Cerro Pirre, 30 jun 1959 (fr), Stern et al. 746 (MO); La Boca de Pirre, 13 oct 1967 (fr), Bristan 1263 (MO). Panamá Barro Colorado Island, Canal Zone, 13 ene 1939 (fl), Zetek 4319 (MO); Barro Colorado Island, Canal Zone, Barbour Trail 350, 26 set 1968 (fr), Croat $6475(\mathrm{MO})$.

4. Smilax spinosa Mill., Gard. Dict. ed. 8, no. 8. 1768. TIPO: MÉXICO. Houston s. $n$. (holotipo, BM).

FIG. 6

Smilax mexicana Griseb. ex Kunth, Enum. P1. 5: 167. 1850. TIPO: MÉXICO. Veracruz; prope Misantla, Schiede \& Deppe 986 (holotipo, B; isotipo: $\mathrm{K}, \mathrm{MO}$ !).

Smilax costaricae Vatke, Linnaea 40: 223. 1876. Smilax mexicana var. costaricae (Vatke) A. DC. Monogr. Phan. 1: 117. 1878. TIPO: CosTA RICA: San José, mayo 1857, Hoffmann 503 \& 504 (sintipo, B, fragmentos e imagen digital US!).

Smilax wagneriana A. DC., Monogr. Phan. 1: 143. 1878. TIPO: PANAMÁ. Chiriquí, abril 1854, $M$.
Wagner 630 (M).

Smilax mazatlanensis Sessé \& Moc., Pl. Nov. Hisp. (ed. 2) 1893. TIPO: MÉXICO: Guerrero: Mazatlán. Sin recolector.

Smilax gaumeri Millsp. ex Gaumer, Field Mus. Nat. Hist., Bot. Ser. 1(4): 357. 1898. TIPO: MÉXICO. Yucatán, Izamal, jun, G.F. Gaumer 687 (holotipo, F; isotipos, G, MO!, imagen digital US! y NY!).

Smilax lundellii Killip \& C. V. Morton, Publ. Carnegie Inst. Wash. 461: 265 1936. TIPO: Guatemala: Petén: recolectado en la Sabana Zis, 3 mayo 1933, C.L. Lundell 3190 (holotipo, US!; isotipo, MICH).

Smilax munda Killip \& C. Morton, Publ. Carnegie Inst. Wash. 461: 265, 1936. TIPO: BRITISH HoNDURAS: on the bank of Río Grande, 23 jul 1933, W.A. Schipp 1181 (holotipo F [foto 52901 $\mathrm{F}$ !]; isotipos, $\mathrm{K}, \mathrm{MICH}, \mathrm{MO}$ ! [fragmentos US!]); syn. nov.

Smilax luculenta Killip \& C. Morton, Publ. Carnegie Inst. Wash. 461: 289 (1936). TIPO: HondurAs. Atlántida: collected near Tela, in thicket near beach, 14 Dec 1927-15 Mar 1928, P.C. Standley 54275 (holotipo US!; isotipo F); syn. nov.

Rizoma con engrosamiento en los nudos. Tallos cilíndricos, glabros, obtusamente angulados en la parte superior e inferior, generalmente en zigzag, armados con fuertes aguijones, aplanados en la parte superior, inermes en la parte inferior. Hojas ovadas a lanceoladas, cartáceas, ocasionalmente algo coriáceas, 4$12.5 \times 5.5-12 \mathrm{~cm}, 5$ nervios desde la base, los nervios primarios prominentes en ambos lados conectados por nervaduras reticuladas, conspicuas, rara vez acúleos en el envés, hojas jóvenes ocasionalmente con manchas blancas o verde pálidas, ápice agudo, base redonda o aguda, margen entero; pecíolos 0.4-2.7 cm de largo. Umbelas solitarias, ocasionalmente racemosas; las estaminadas con pedúnculo aplanado, 1.5-6 mm, más corto que el pecíolo subyacente; tépalos $2 \mathrm{~mm}$, anteras $0.5 \mathrm{~mm}$, filamentos $0.7-1 \mathrm{~mm}$, más largos que las anteras; las pistiladas con pedúnculo aplanado, 2$26 \mathrm{~mm}$; tépalos ca. $2 \mathrm{~mm}$. Bayas 4-7 mm de diámetro, rojas, moradas o negras.

DISTRIBUCIÓN Y HÁBITAT. Es un bejuco que posee 
A
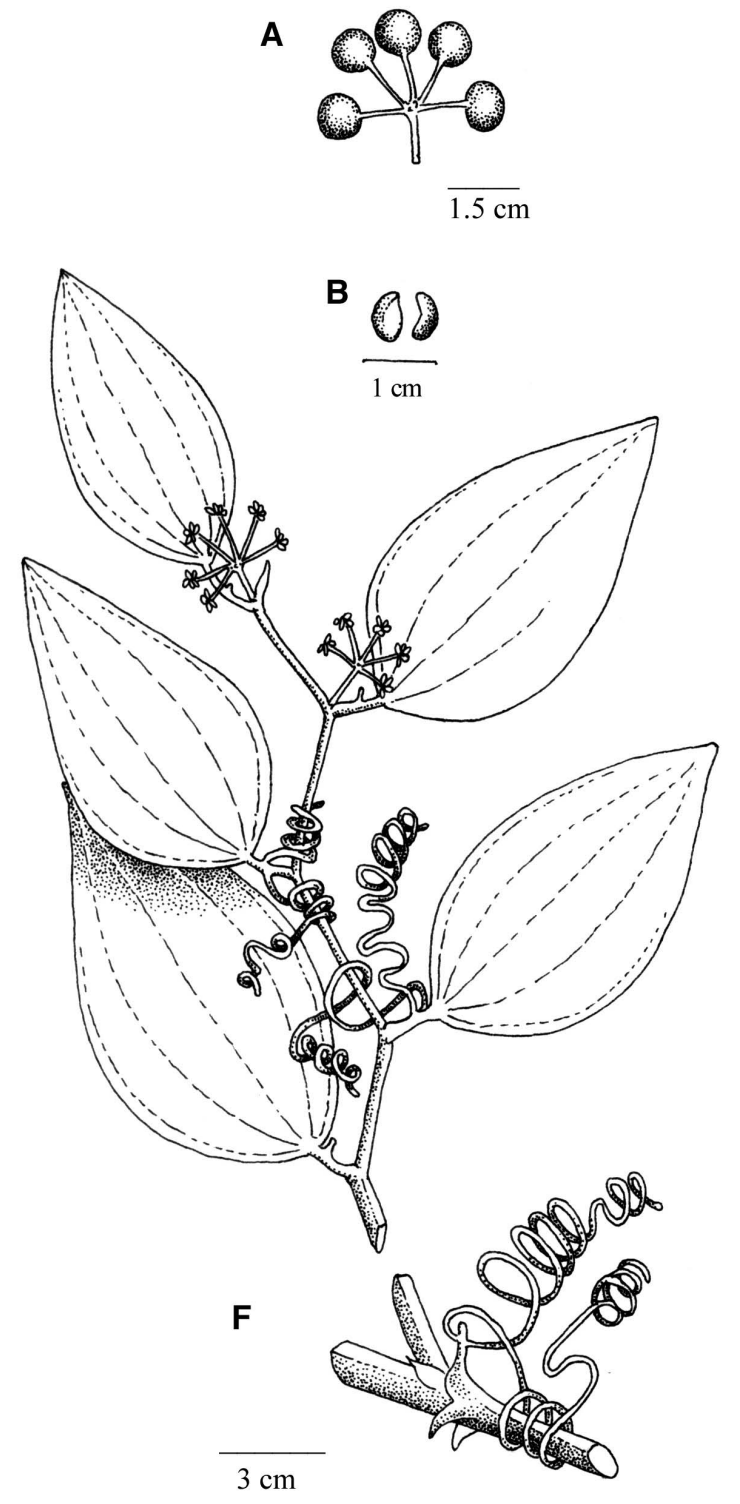
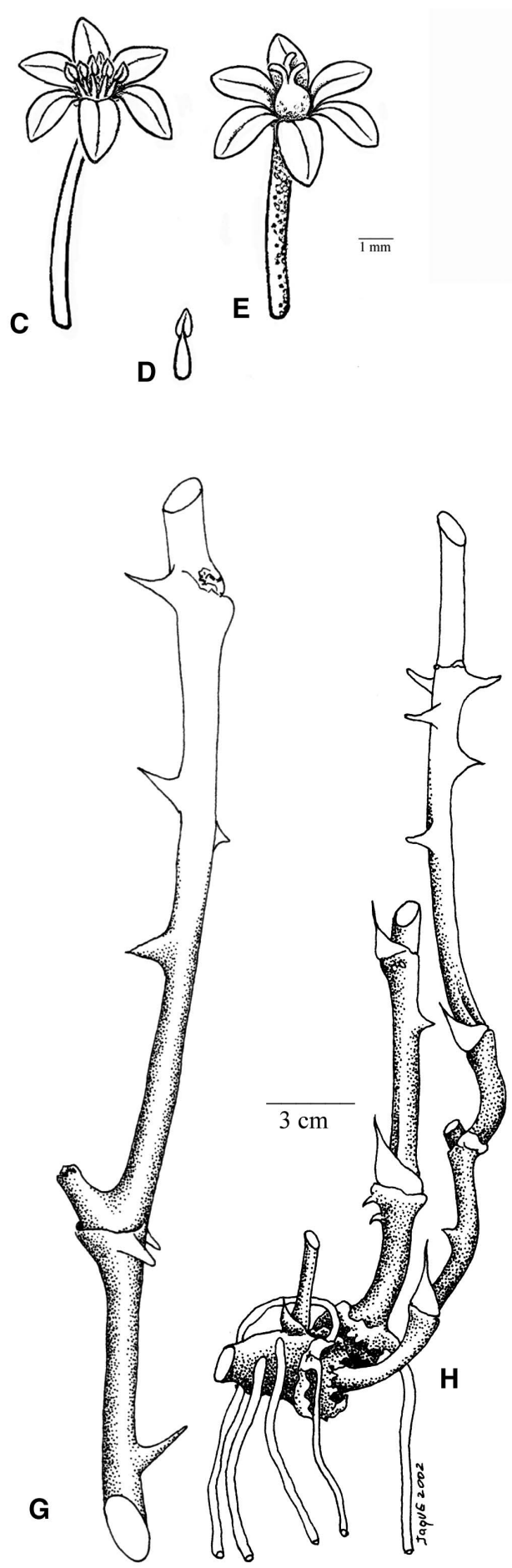

Figura 6. Smilax spinosa Mill. A. Frutos, B. Semillas, C. Flor masculina, D. Estambre, E. Flor femenina, F. Rama fértil con flores, G. Tallo, H. Tallo y rizoma. 


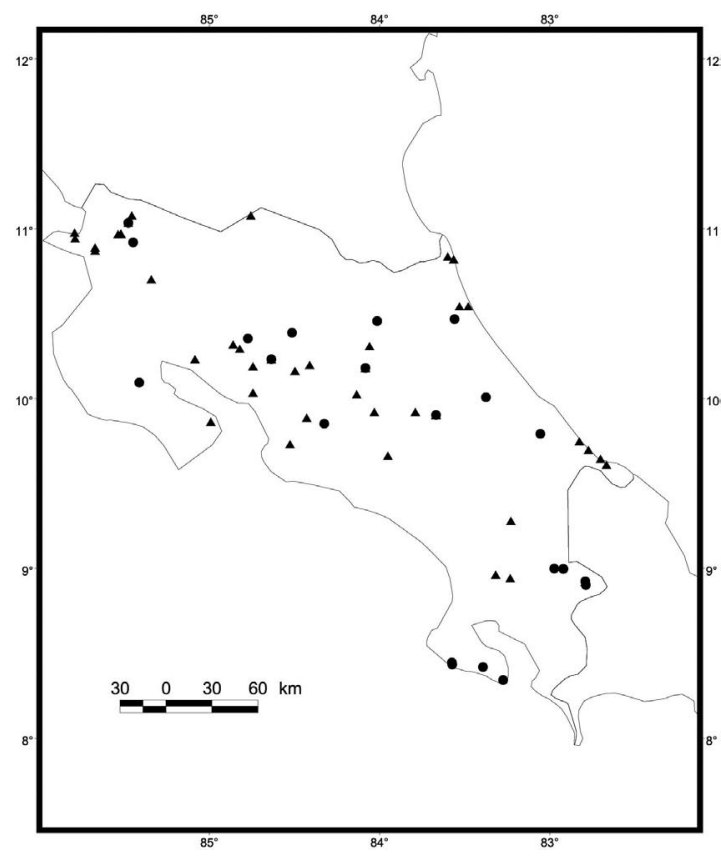

Figura 7. Distribución geográfica de Smilax panamensis ( • ) y $S$. spinosa ( $\bullet$ ) en Costa Rica.

una distribución amplia. Se encuentra en bosques secos, húmedos y premontanos, entre 0 y $2000 \mathrm{~m}$ (Fig. 7). Se distribuye desde el norte de México hasta Panamá (Huft 1994).

NOMBRES COMUNES Y USOS. Zarzaparrilla, cuculmeca, bejuco de corona. El rizoma es usado para los riñones, enfermedades venéreas, como regulador menstrual, para la limpieza después del parto, flujo blanco y fertilidad de la mujer (House et al. 1994). En El Salvador se usa para hacer las coronas de Jesús Nazareno, bajo el sinónimo de $S$. mexicana (Choussy 1926).

Smilax spinosa se caracteriza por los tallos obtusamente angulados, en zigzag, con manchas rojas oscuras, aguijones presentes, hojas con acúleos en el envés, hojas jóvenes manchadas de color verde pálido o blancas, pedúnculos iguales o más cortos que el pecíolo subyacente, tépalos $c a .2 \mathrm{~mm}$ y bayas negras o moradas.

Smilax luculenta fue descrita con base en material vegetativo. Se distingue de otras especies de Centro América por sus hojas coriáceas con base cordada, sin aguijones, nervios prominentes, nervaduras secundarias oscuras y pedúnculos más cortos que los pecío- los. Sin embargo, su amplia variabilidad morfológica y su distribución coinciden con las de $S$. spinosa, por lo que aquí se considera sinónimo.

La morfología de las hojas ha sido un carácter discutido por muchos autores. Un bejuco puede mostrar una amplia variabilidad en la forma de las hojas, que depende de la posición de las yemas foliares, de la edad o del grado de madurez de la hoja, hojas fértiles versus estériles y de los factores ambientales como el suelo o la sombra (Gaskin \& Berry 1998). Una variación fenotípica considerable en las estructuras vegetativas tambien se observa en $S$. domingensis.

ESPECÍMENES EXAMINADOS. Belice. Cayo: Between the Western Highway and the Sibun River, $c a .7 \mathrm{~km}$ west of intersection of Western Highway and coastal road "Manatee Highway", $17^{\circ} 16^{\prime} \mathrm{N}, 88^{\circ} 34^{\prime} \mathrm{W}, 10-50 \mathrm{~m}, 13$ jul 1995 (fl), Atha et al. 1114 (MO). Toledo: Río Mojo, mouth to 8 miles upstream, 8 ago 1975 (fl), Dwyer \& Coomes 12947 (MO). Costa RicA. Alajuela: Los Chiles, orilla del Río Frío, ca. 2 $\mathrm{km}$ aguas arriba de Los Chiles, $10^{\circ} 01^{\prime} 35^{\prime \prime} \mathrm{N}, 84^{\circ} 43^{\prime} 15^{\prime \prime} \mathrm{W}, 40-$ 50 m, 18 ago 2001 (fr), Ferrufino et al. 150 (USJ); San Ramón, $10 \mathrm{~km}$ NNW of San Ramón by road on way to San Lorenzo, $2.5 \mathrm{~km} \mathrm{~S}$ of Balsa, remnant of primary forest, $10^{\circ} 09^{\prime} \mathrm{N}, 84^{\circ} 29^{\prime} \mathrm{W}, 1200 \mathrm{~m}, 25$ abr 1983 (fl), Liesner \& Judziewicz 15005 (CR, MO); Upala, Bijagua, El Pilón, Río Celeste, margen izquierda, aguas arriba a partir del puente camino a La Laguna del Roble, 24 abr 1988 (fl), Herrera 1884 (CR); Llanura Bonita de Zarcero, 1600 m, 6 abr 1938 (fl, fr), Smith 602 (MO); Near San Ramón on the calle Los Angeles, $10^{\circ} 86^{\prime} \mathrm{N}, 84^{\circ} 27^{\prime} \mathrm{W}, 1100 \mathrm{~m}, 27$ mar 1982 (fl), Barringer et al. 2154 (MO); Monteverde Reserve, Atlantic slope, Río Peñas Blancas valley, 960 m, 19 nov 1984 (fr), Haber 1025 (MO). Cartago: Turrialba, Platanillo, 22 abr 1951 (fr), León 3355 (USJ); Turrialba, terrenos del Instituto Interamericano de Ciencias Agrícolas, 600 m, 14 oct 1949 (f1), León 1838 (USJ). Heredia: Barva, 1170 m, 20 dic 1940 (fl), León 448 (CR); Parque Nacional Braulio Carrillo, Cantón de Sarapiquí, Puesto El Ceibo, W of Río Guácimo, off trail that runs due $\mathrm{E}$ from Transect Trail at $730 \mathrm{~m}$, $10^{\circ} 17^{\prime} 27^{\prime \prime} \mathrm{N}, 84^{\circ} 03^{\prime} 38^{\prime \prime} \mathrm{W}, 760 \mathrm{~m}, 22$ abr 1994, Boyle 3145 (INB, MO). Guanacaste: Abangares, Cebadilla, 1400 m, 21 jul 1985 (fr), Haber \& Bello 2250 (CR, MO); Cañas, Paso Hondo, en la berna de un canal de riego, 23 abr 1992 (fl), Rojas s.n. (USJ); La Cruz, Parque Nacional Santa Rosa, Península de Santa Elena, Murciélago, alrededor del aeropuerto, $10^{\circ} 54^{\prime} 10^{\prime \prime} \mathrm{N}, 85^{\circ} 43^{\prime} 50^{\prime \prime W}, 50 \mathrm{~m}, 10$ set 1994 (fr), Cano 136 (CR, INB, MO); La Cruz, Santa Rosa National Park, evergreen forest (bosque húmedo), $2 \mathrm{~km}$ east of park headquarter, $10^{\circ} 51^{\prime} \mathrm{N}, 85^{\circ} 37^{\prime} \mathrm{W}, 200-300 \mathrm{~m}, 23$ jun 1977 (fl), Liesner \& Lockwood 2410 (CR, MO); Liberia, Parque Nacional Guanacaste, Estación Cacao, $10^{\circ} 55^{\prime} 45^{\prime \prime} \mathrm{N}, 85^{\circ} 28^{\prime} 15^{\prime \prime} \mathrm{W}, 1100$ m, 31 oct 1990 (fr), Chávez et al. 329 (INB); Parque Nacional Guanacaste, Estación Pitilla, Sendero El Mismo, Finca La Pasmompa, $11^{\circ} 02^{\prime} 00^{\prime \prime N}, 85^{\circ} 24^{\prime} 30^{\prime \prime} \mathrm{W}, 700$ m, 9 dic 1990 (fl), 
Ríos 254 (CR, INB, MO). Limón: Parque Nacional Tortuguero, $2 \mathrm{~km}$ al sur de Tortuguero, bosque inundable a la orilla de la laguna, $10^{\circ} 31^{\prime} \mathrm{N}, 83^{\circ} 30^{\prime} \mathrm{W}, 3 \mathrm{~m}, 4$ nov 1988 (fr), Robles 2169 (CR, MO); Puerto Vargas, Parque Nacional Cahuita, 3 set 1978 (fr), Gómez-Laurito 3953 (USJ); Talamanca, Puerto Vargas, $1 \mathrm{~km}$ por el camino de entrada, $9^{\circ} 42^{\prime} 03^{\prime \prime N}, 82^{\circ} 49^{\prime} 06^{\prime \prime W}, 2-3$ m, 24 may 1995, Gómez-Laurito et al. 12789 (USJ). Puntarenas: Buenos Aires, Reserva Indígena Boruca, Fila Moras, camino a Boruca, $8^{\circ} 599^{\prime} 30^{\prime \prime} \mathrm{N}$, $83^{\circ} 20^{\prime} 40^{\prime \prime} \mathrm{W}, 800 \mathrm{~m}, 7$ mar 1993 (fl), Rojas \& Zúñiga 172 (CR, INB, MO); Las Alturas Biological Station, about $100 \mathrm{~m}$ into forest from pasture side trail, premontane wet forest, $8^{\circ} 57^{\prime} 05^{\prime \prime} \mathrm{N}, 82^{\circ} 50^{\prime} 15^{\prime \prime} \mathrm{W}, 1450 \mathrm{~m}, 9$ jul 1998 (fr), Boyle \& Alford 5208 (CR, USJ); Península de Nicoya, Curú, Pozo Colorado Trail, on ridge toward Punta Quesera, mangroves at Río Curú mouth, $9^{\circ} 46-48^{\prime} \mathrm{N}, 84^{\circ} 54-58^{\prime} \mathrm{W}, 0-200 \mathrm{~m}, 30$ ago 1995 (fr), Sanders et al. 17722 (CR, USJ); Puntarenas, Cordillera de Tilarán, San Luis, por el playón del Río San Luis, Monteverde, $10^{\circ} 16^{\prime} 33^{\prime \prime} \mathrm{N}, 84^{\circ} 47^{\prime} 45^{\prime \prime} \mathrm{W}, 1100 \mathrm{~m}, 18 \mathrm{abr}$ 1994 (fl), Fuentes 729 (INB); Monteverde, altos de Lindora, Vertiente Pacífica, $10^{\circ} 18^{\prime} \mathrm{N}, 84^{\circ} 50^{\prime} \mathrm{W}, 1300 \mathrm{~m}, 7$ dic 1988 (fr), Bello 579 (CR, MO); Buenos Aires, R. I. Ujarrás, Cordillera de Talamanca, Ujarrás, margen izquierda de Quebrada Dodora, camino a Río Lori, $09^{\circ} 17^{\prime} 50 " \mathrm{~N}$, 8315'30"W, 1500 m, 12 mar 1993 (fl), Fernández 621 (INB, MO). San José: Ciudad Colón, Mora, bosque regenerado, $c a$. 35 años, 800 m, 18 feb 2000, Morales 1406 (USJ); Curridabat, San Francisco, ene 1980 (fr), Stiles s.n. (USJ); Dota, Cordillera de Talamanca, Madreselva, orilla de bosque, $9^{\circ} 40^{\prime} 05^{\prime \prime N}, 83^{\circ} 57^{\prime} 22^{\prime \prime} \mathrm{W}, 2500-2600$ m, 24 ago 1996 (fr), Gómez-Laurito 12877 (USJ); Mora, Tabarcia, Alto de Vargas, borde de bosque, 920 m, 22 abr 1963 (fl), Jiménez 662 (CR); Zona Protectora Cerros de Turrubares, Las Delicias, Sitio

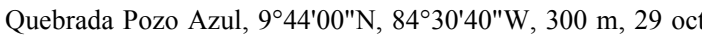
1991 (fr), Zúñiga 525 (USJ). El SALVADOR. Ahuachapán: San Francisco Menéndez, El Corozo, Mariposario, zona baja "Los Peralta", $13^{\circ} 49^{\prime} \mathrm{N}, 89^{\circ} 59^{\prime} \mathrm{W}, 380 \mathrm{~m}, 14$ abr 2000 (fl), Rosales 589 (MO). San Miguel: Volcán Conchagua, 1316'35"N, 8748'50"W, 400 m, 31 ene 1998 (fr), Monro et al. 2142 (MO). Guatemala. Izabal: Río Dulce, 1 m, 27 jul 1988 (bo, fl), Martínez et al. 23108 (MO). Petén: Forest edge at oficina de "Cerro Cauhui", 26 abr 1986 (fl), Walker 1284 (MO). HoNDURAS. Atlántida: La Ceiba, on the mountain slopes and coastal plains, 4 ago, 1938 (fl, fr), Yuncker et al. 8812 (MO) Francisco Morazán: Quebrada La Orejona, lado E de la UNAH, 1000 m, 23 abr 1982 (fl), Belibasis 197 (MO). MÉXICO. Campeche: Champotón, carretera ChampotónUlumal, Río Champotón donde lo cruza el puente entre El Zapotal y Ulumal, $0.5-1 \mathrm{~km}$ al S de El Zapotal, $19^{\circ} 16^{\prime} 55^{\prime \prime} \mathrm{N}$, 90³6'50"W, 0-50 m, 6 jun 1997 (fl), Carnevali et al. 4489 (MO). Oaxaca: Chiltepec, 13 m, 24 abr 1967 (fl), Martínez 1376 (MO). Nicaragua. Chontales: $5.6 \mathrm{~km} \mathrm{~S}$ of La Libertad W slope of Cerro El Gallo, ca. $12^{\circ} 10^{\prime} \mathrm{N}, 85^{\circ} 10^{\prime} \mathrm{W}$, 440-480 m, 7 jun 1980 (fl, fr), Stevens et al. 17504 (MO). Granada: Laguna de Apoyo “ Babilonia”, $11^{\circ} 55^{\prime} \mathrm{N}, 86^{\circ} 04^{\prime} \mathrm{W}$, 40-80 m, 30 may 1981 (fl), Moreno \& Henrich 890 (MO). Matagalpa: Behind La Selva Negra Hotel, slopes of Cerro Picacho, near the border with depto Jinotega, $13^{\circ} 00^{\prime} \mathrm{N}$,
855' 'W, 1200-1540 m, 23-25 may 1985 (fl), Davidse et al. 30425 (MO). Panamá. Chiriquí: km 4.6 on Volcancito road W of Boquete, $4600 \mathrm{ft}$., 14 may 1971 (fl), Proctor 31874 (MO). Los Santos: Guayabo, a place several miles W of Tonosí, 1 mar 1963 (bo, fl), Stern et al. 1899 (MO). Panamá: Barra Colorado Island, Canal Zone, end of Peña Blanca Península, central cove, 10 may 1968 (fl), Croat 5449 (MO); Barra del Colorado, N side, between town and ocean beach, $10^{\circ} 47^{\prime} \mathrm{N}, 83^{\circ} 35^{\prime} \mathrm{W}, 0-2 \mathrm{~m}, 26$ ene 1986 (fr), Stevens 24097 (MO); margin of Laguna de atras, between Barra del Colorado and the mounth of the Río San Juan, $10^{\circ} 48^{\prime}$ y $10^{\circ} 52^{\prime} \mathrm{N}, 83^{\circ} 37^{\prime}$ y $83^{\circ} 38^{\prime} \mathrm{W}, 5 \mathrm{~m}, 21$ set 1986 (fl), Davidse \& Herrera 31519 (MO). Veraguas: Pasture, fencerow and roadside just $\mathrm{S}$ of Santa Fe, ca. 450 m, 17 nov 1973 (fr), Nee 8013 (MO).

5. Smilax spissa Killip \& C. Morton, Publ. Carnegie Inst. Wash. 461: 273. 1936. TIPO: CosTA RiCA. Entre La Muerte y División, 19 ene 1891. H. Pittier 3470 (holotipo, US!).

FIG. 8

Rizoma tuberoso, rojo. Tallos cilíndricos, glabros, armados en la parte inferior con aguijones robustos, inerme en la parte superior. Hojas oblongas a lanceoladas, cartáceas 7-22 × 3-9 cm, 5 nervios desde la base, a veces trinervada, los nervios primarios externos submarginales, algo prominentes en el envés, conectados por nervaduras paralelas, ápice acuminado a cuspidado, base aguda, margen entero; pecíolos 1-2 cm de largo. Umbelas solitarias; las estaminadas con pedúnculo subcilíndrico, $2-4.5 \mathrm{~cm}$, igual o más largo que el pecíolo subyacente; tépalos 5-6 mm, filamentos 1-1.5 mm, anteras 1.5-2 mm, más largos que los filamentos; las pistiladas con pedúnculo subcilíndrico, 1.5-2.5 cm, más largo que el pecíolo subyacente; tépalos ca. $4 \mathrm{~mm}$. Bayas 7-12 mm de diámetro, rojas.

DISTRIBUCIÓN Y HÁBITAT. Se encuentra en bosques húmedos del Pacífico Central y Pacífico Sur del país, entre 0 y 900 m (Fig. 9). Se distribuye en Costa Rica y Panamá.

Nombres COMUNES. Cuculmeca, cuculmeca roja.

Smilax spissa se caracteriza por el rizoma tuberoso, tallos cilíndricos con aguijones pequeños, hojas con 5 nervios desde la base conectados por nervaduras paralelas, pedúnculo más largo que el pecíolo subyacente, tépalos ca. $4 \mathrm{~mm}$ y bayas rojas.

Killip \& Morton (1936) anotan que esta especie ha sido confundida con $S$. panamensis, pero ambas tienen características importantes que las distinguen. 


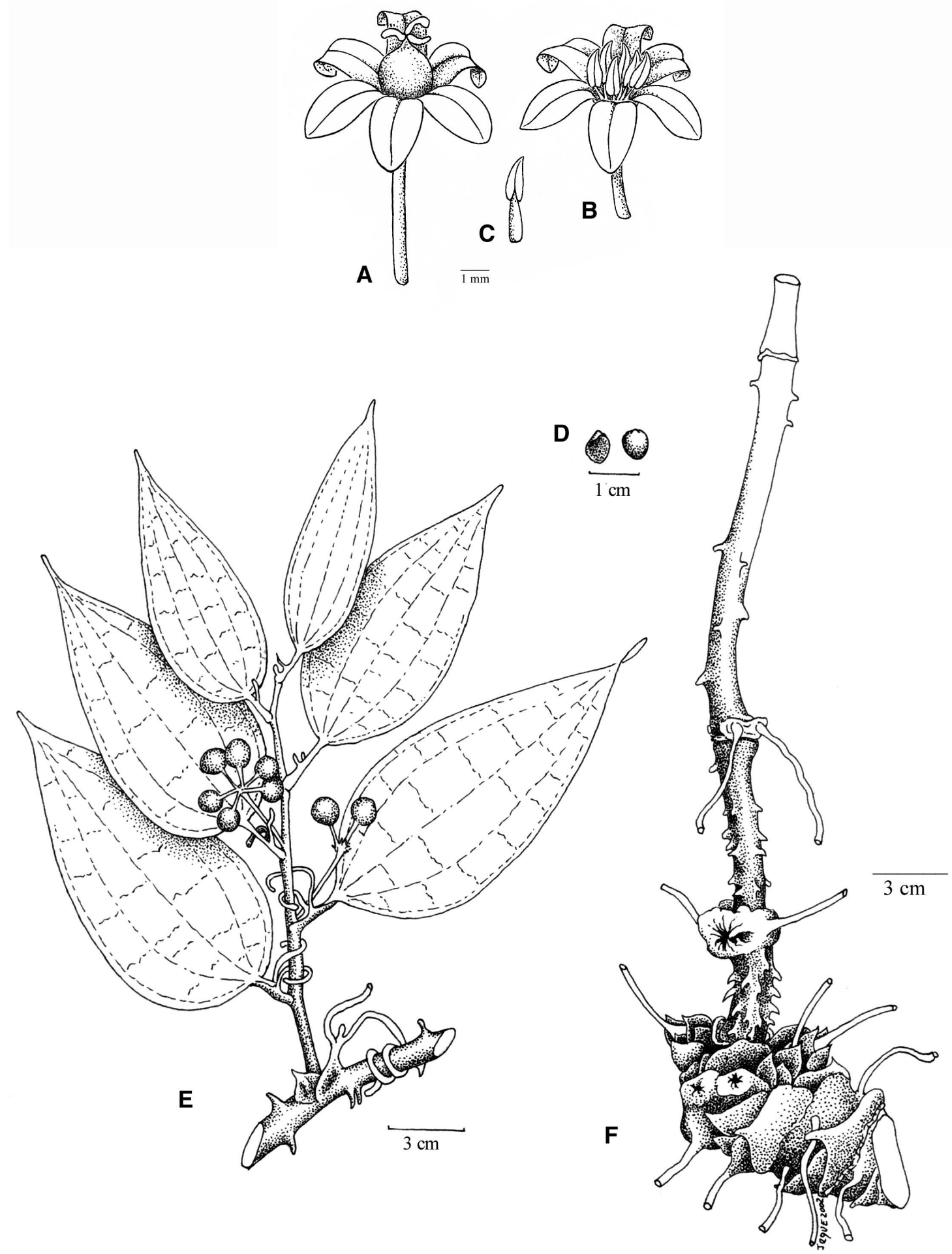

Figura 8. Smilax spissa Killip \& C. Morton. A. Flor femenina, B. Flor masculina, C. Estambre, D. Semillas, E. Rama fértil con frutos, F. Tallo y rizoma. 


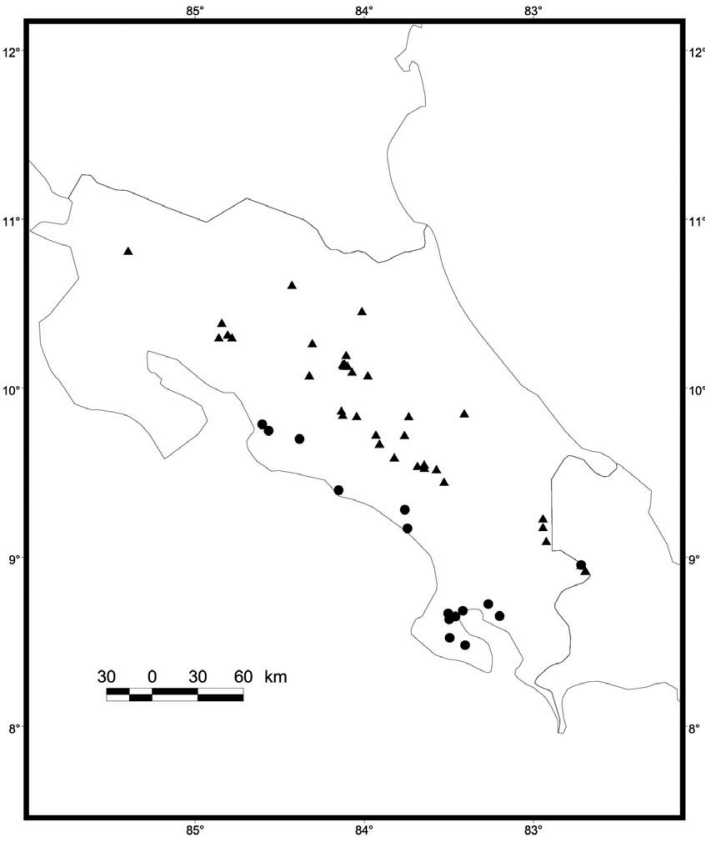

Figura 9. Distribución geográfica de Smilax spissa (•) y S. subpubescens ( $\mathbf{\Delta}$ ) en Costa Rica.

Smilax spissa también ha sido confundida con $S$. subpubescens por los pedúnculos en relación a los pecíolos, y con $S$. domingensis por las bayas rojas. En los herbarios de Costa Rica, Panamá y MO se encuentran en su mayoría especímenes con botones florales, frutos maduros, o rara vez flores abiertas. Todavía es necesario recolectar ejemplares con flores de ambos sexos, para observar un mayor número de muestras.

ESPECÍMENES EXAMINADOS. COSTA RICA. Puntarenas: Cordillera de Talamanca, trail between headwater on the Río Bella Vista and Sitio Cotón (Cotonsito) on the Río Cotón, $9^{\circ} 49^{\prime} \mathrm{N}-9^{\circ} 57^{\prime} \mathrm{N}, 82^{\circ} 46^{\prime} \mathrm{W}-82^{\circ} 49^{\prime} \mathrm{W}, 1800-2200 \mathrm{~m}, 11 \mathrm{mar}$ 1984 (fr), Davidse 25524 (CR, MO); Foothills of the Cordillera de Talamanca, Sitio Coto Brus, $c a .8^{\circ} 59^{\prime} \mathrm{N}$ $82^{\circ} 46^{\prime} \mathrm{W}, 1800-1900 \mathrm{~m}, 3$ set 1983 (fr), Davidse 24516 (CR, MO); Punta Catedral, ca. $7 \mathrm{~km} \mathrm{SE}$ of Quepos, $9^{\circ} 22.5^{\prime} \mathrm{N}$, 840' W, 20-70 m, 20 ago 1985 (fr), Grayum \& Sleeper 5900 (CR, MO); Golfito, Parque Nacional Corcovado, Península de Osa, Bonanza, $8^{\circ} 31^{\prime} 30^{\prime \prime} \mathrm{N}, 83^{\circ} 25^{\prime} 40^{\prime \prime} \mathrm{W}, 200-300 \mathrm{~m}, 5 \mathrm{mar}$ 1997 (bo), Azofeifa 256 (INB); Puntarenas, Punta Banco, 0200 m, 22 ago 1988 (fr), Chavarría et al. 259 (USJ); Reserva Biológica Carara, Lomas Pizote, sendero a Bijagual, 9०47'10"N, 84³5'10"W, 300 m, 8 dic 1989 (fr), Jiménez \& Zúñiga 761 (INB, USJ); Reserva Forestal Golfo Dulce, Aguabuena, Sector Cuenca oeste, $8^{\circ} 42^{\prime} 20^{\prime \prime} \mathrm{N}, 83^{\circ} 31^{\prime} 30^{\prime \prime} \mathrm{W}$, 50-150 m, 22 oct 1991 (fr), Aguilar 600 (CR, MO); Osa, Faja Costeña de la Bahía de Coronado, Uvita, camino a San Josecito, paralelo a Río Morete (Río Higuerón en el mapa), $9^{\circ} 11^{\prime} 30^{\prime \prime N}, 83^{\circ} 45^{\prime} 25^{\prime \prime} \mathrm{W}, 100 \mathrm{~m}$, Aguilar et al. 1533 (INB, MO). San José: Puriscal, Zona Protectora La Cangreja, Cuenca del Tulín, Falda Sur, Fila Cangreja, 9 $9^{\circ} 2^{\prime} 10^{\prime \prime N}$, $84^{\circ} 22^{\prime} 25^{\prime \prime W}, 650$ m, 7 feb 1997 (bo), Jiménez 2229 (INB); Tarrazú, San Lorenzo, camino entre Cerro Pito y Cerro Toro, rumbo a Quepos, 9०17'54"N, 8346'20"W, 600-700 m, 26 may 1998 (fr), Valverde 972 (CR, USJ); Western part of Montañas Jamaica, $c a .3 \mathrm{~km}$ NE of Bijagual de Turrubares, Carara Reserve, $9^{\circ} 45.5^{\prime} \mathrm{N}, 8^{\circ} 33^{\prime} \mathrm{W}, 500-600 \mathrm{~m}, 7$ ago 1985 (fr), Grayum et al. 5843 (CR, MO). PANAmÁ. Coclé: Between Continental Divide above El Cope and El Petroso sawmill and the Río Blanco to the $\mathrm{N}$ a $5 \mathrm{hr}$ hike, $8^{\circ} 38^{\prime} \mathrm{N}, 80^{\circ} 36^{\prime} \mathrm{W}, 1400$ ft., 13 dic 1980 (fr), Sytsma et al. 2398 (MO); Los Pedregales, ridge between Río Blanco del Norte and Río Caño Sucio, ridge eventually leads to Cerro Tife and Continental Divide, $8^{\circ} 44^{\prime} \mathrm{N}, 81^{\circ} 40^{\prime} \mathrm{W}, 500 \mathrm{~m}, 22$ feb 1982 (bo), Knapp \& Dressler 3811 (MO). Panamá: Barro Colorado Island, Shannon Trail 700, 5 jul 1971 (fr), Croat 15260 (MO). San Blas: Nusagandi, trail from camp NW to a Quebrada, $9^{\circ} 19^{\prime} \mathrm{N}$, $78^{\circ} 15^{\prime} \mathrm{W}, 300$ m, 31 jul 1984 (fr), Nevers \& León 3592 (MO).

6. Smilax subpubescens A. DC. in A. DC. \& C. DC., Monogr. Phan. 1: 69. 1878. TIPO: MÉXICO. Bourgeau 2578 (holotipo, P, foto $40299 \mathrm{~F}$ !).

FIG. 10

Smilax calocardia Standl., Publ. Field Mus. Nat. Hist., Bot. Ser. 22(1): 7. 1940. TIPO: PANAMÁ. Chiriquí: Volcán de Chiriquí, alt. 2250 m, ago 1938, M.E. Davidson 953 (holotipo, F; isotipo, MO!).

Smilax rufa Lundell, Contr. Univ. Michigan Herb. 7: 4. 1942. TIPO: MÉXICO. Chiapas, cerca del Porvenir, 3200 m, 6 jul 1941, Matuda 4591 (holotipo, MICH; isotipo, LL).

Smilax venosa Lundell, Wrightia 3(8): 165, f. 56 1966. TIPO: MÉXICO. Chiapas: Pinabeto, cerca de Montozintla, alt. 2585 m, 7 may 1945, Matuda 5426 (holotipo: LL, foto $61375 \mathrm{~F}$ !).

Rizoma con engrosamiento en los nudos. Tallos obtusamente cuadrangulares, inermes, rojo-tomentosos, glabros con la edad o a veces persistentemente tomentosos. Hojas ovadas, láminas glabras en el haz, rojo-tomentosas en el envés y las vainas; glabras con la edad o raramente con pubescencia persistente, cartáceas a subcoriáceas, $10-16 \times 5.5-10.5 \mathrm{~cm}, 5-7$ nervios desde la base, los nervios primarios prominentes en el envés, el par exterior submarginal, conectados por nervaduras reticuladas, conspicuas; ápice acuminado, base cordada, redonda o truncada, margen entero; pecíolos 1.5-5 cm de largo. Umbelas solitarias; las estaminadas con pedúnculo aplanado, 


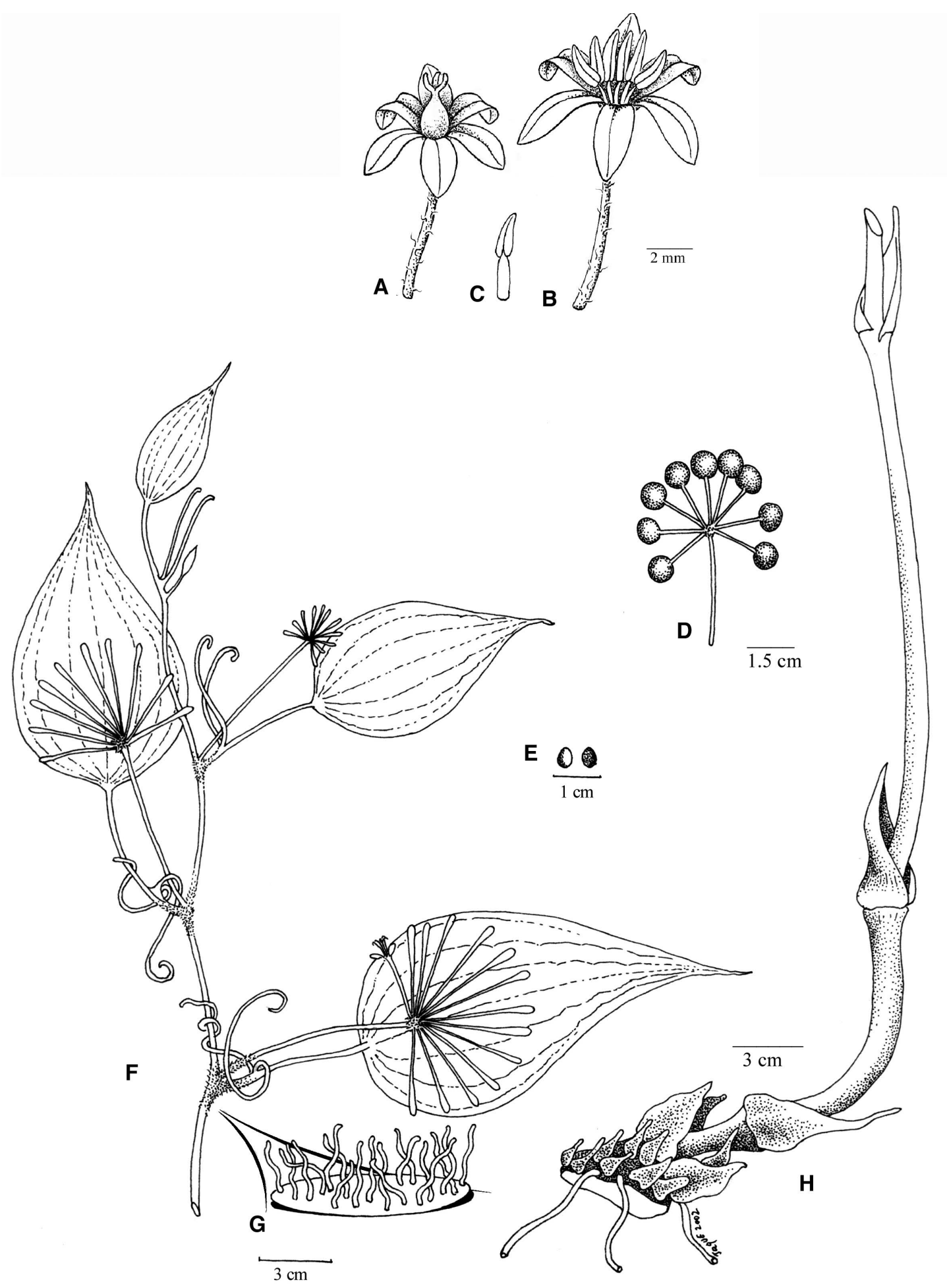

Figura 10. Smilax subpubescens A. DC. A. Flor femenina, B. Flor masculina, C. Estambre, D. Frutos, E. Semillas, F. Rama fértil con flores, G. Tricomas de la base del pecíolo, H. Tallo y rizoma. 
1.3-4 cm, generalmente más corto que el pecíolo subyacente, ocasionalmente pubescente cerca de la base; tépalos 4-6 mm, filamentos 2.5-3 mm, anteras $c a$. 2 $\mathrm{mm}$, más cortas que los filamentos; las pistiladas con pedúnculo aplanado, $1.5-5 \mathrm{~cm}$, más corto o más largo que el pecíolo subyacente; tépalos 3-4 mm. Bayas 5-7 $\mathrm{mm}$, anaranjadas.

DISTRIBUCIÓN Y HÁBITAT. Se halla en bosques húmedos montanos y bosques nublados, entre 1300 y 3000 m (Fig. 9). Se distribuye desde México hasta Panamá.

Nombres comunes y usos. Bejuco canasta. El tallo se usa para hacer canastos y amarres.

Smilax subpubescens se caracteriza por los tallos cilíndricos sin aguijones, con pubescencia tomentosa roja en las partes jóvenes, entrenudos y base del pecíolo, glabros en la madurez, hojas ovadas a lanceoladas, glabras, con base cordada, tépalos $c a .4$ $\mathrm{mm}$, pedúnculos iguales o más largos que el pecíolo subyacente y bayas anaranjado brillante. Es un bejuco que crece en bosques nublados a elevaciones altas.

Los especímenes glabros de esta especie han sido confundidos con $S$. domingensis, $S$. panamensis y $S$. spissa. Killip \& Morton (1936) mencionan que $S$. tomentosa Kunth es una especie de Sudamérica, relacionada con esta especie, que se diferencia por la presencia de 3 estaminodios en las flores pistiladas y las hojas densamente pubescentes. Sin embargo, es una especie muy cercana a $S$. mollis y es probable que no sea distinta a ésta.

También De Candolle (1878) describe S. subpubescens con pequeños aguijones en el tallo. En su revisión taxonómica, Killip \& Morton (1936) afirman que el tipo y otros especímenes examinados no presentan aguijones, por lo que se espera no encontrar aguijones en $S$. subpubescens.

ESPECÍMENES EXAMINADOS. Costa Rica. Alajuela: Grecia, Bosque del Niño, Reserva Forestal de Grecia, 16001800 m, 18 mar 1987 (fl), Umaña \& Gómez 163 (CR); Reserva Forestal El Chayote, orilla de camino, 2000 m, 29 set 1992 (fr), Sánchez et al. 250 (USJ); San Carlos, Fila Volcán Viejo, $1800-2000 \mathrm{~m}, 10^{\circ} 15^{\prime} \mathrm{N}, 8^{\circ} 18^{\prime} \mathrm{W}, 13$ nov 1986 (fr), Gómez-Laurito 11248 (USJ); entre el cráter y la Laguna Fría del Volcán Poás, en bosquecillo intrincado, 2600 m, 13 ago 1964 (fl), Jiménez 2267 (CR, MO). Cartago: Paraíso, P. N. Tapantí, Valle del Reventazón, 0943'35"N, 8346'28"W, 1450 m, 31 mar 1995 (fr), Morales \& Bohs 3828 (INB, MO).
Guanacaste: Abangares, $5 \mathrm{~km}$ N Monteverde at Río Negro on road to Finca San Bosco, Atlantic slope, $10^{\circ} 22^{\prime} \mathrm{N}, 84^{\circ} 49^{\prime} \mathrm{W}$, 1300 m, 2 feb 1989 (fr), Haber \& Zuchowski 9067 (CR, MO); Liberia, Parque Nacional Rincón de la Vieja, Cordillera de Guanacaste, Río Blanco, Estación Las Pailas, $10^{\circ} 46^{\prime} 40 " \mathrm{~N}$, $85^{\circ} 21^{\prime} 05^{\prime \prime} \mathrm{W}, 800 \mathrm{~m}, 27$ nov 1992 (fr), Cano et al. 29 (INB). Heredia: Parque Nacional Braulio Carrillo, Estación Barva, bosque primario y potreros, $10^{\circ} 07^{\prime} 22^{\prime \prime} \mathrm{N}, 84^{\circ} 07^{\prime} 15^{\prime \prime} \mathrm{W}, 2500$ m, 20 jun 1990 (fl), Varela 63 (CR, INB, MO); Sarapiquí road, Catarata Los Angeles, 1400 m, 8 oct 1969 (fr), Schnell 1082 (MO); Barva, Parque Nacional Braulio Carrillo,Volcán Barva, $10^{\circ} 07^{\prime} 20^{\prime \prime} \mathrm{N}, 8^{\circ} 06^{\prime} 00^{\prime \prime} \mathrm{W}, 2600 \mathrm{~m}, 7$ ago 1989 (fl), Rivera 29 (INB, MO); Cerros de Zurquí, northeast of San Isidro, 2000-2400 m, 3 mar 1926 (fl), Standley \& Valerio 50770 (US). Limón: Cordillera de Talamanca, atlantic slope, Kamuk massif, ridge between the Río Tararia and the NE most Kamuk paramo, $9^{\circ} 15^{\prime}-9^{\circ} 16^{\prime} \mathrm{N}, 82^{\circ} 59^{\prime} \mathrm{W}, 2400-2900 \mathrm{~m}, 17$ set 1984 (fl), Davidse \& Herrera 29262 (MO). Puntarenas: Coto Brus, Zona Protectora Las Tablas, Cuenca Térraba-Sierpe, Sitio Tablas, bosque primario, $8^{\circ} 57^{\prime} 03^{\prime \prime} \mathrm{N}, 82^{\circ} 44^{\prime} 38^{\prime \prime} \mathrm{W}, 1850$ m, 25 jul 1997 (fl), Navarro 766 (INB); Reserva Biológica Monteverde, Pacific slope, road from field station to continental divide and La Ventana, $10^{\circ} 18^{\prime} \mathrm{N}, 84^{\circ} 47^{\prime} \mathrm{W}, 1500-1600 \mathrm{~m}$, 11 jun 1992 (fl), Haber \& Stevenson 11202 (INB). San José: Dota, Cordillera de Talamanca, La Cima de Copey de Santa María de Dota, 9०40'35"N, 8355'00"W, 1000 m, 7 jun 1989 (fl), Chavarría 402 (CR, INB, MO); La Palma, dic 1919, Jiménez 1189 (CR); Pérez Zeledón, Parque Internacional La Amistad, Cordillera de Talamanca, entre las nacientes de las Quebradas Barranca y Río Blanco, Finca San Carlos, $9^{\circ} 31^{\prime} 47^{\prime \prime N}, 83^{\circ} 35^{\prime} 30^{\prime \prime} \mathrm{W}, 2350$ m, 5 abr 1995 (fl), Aguilar \& Garrote 3852 (CR, INB, MO); Southwest slope of Cerro Francisco, along the road from Santa María de Dota to El Empalme, $1 \mathrm{~km}$ southeast of Jardín, $2150 \mathrm{~m}, 21$ may 1991 (fr), Grant \& Ramírez 9101518 (CR, US); Vásquez Coronado, Parque Nacional Braulio Carrillo, trail from highway at La Ventana to Bajo La Hondura, $10^{\circ} 04^{\prime} \mathrm{N}, 83^{\circ} 59^{\prime} \mathrm{W}, 1100-1300$ m, 4 set 1990 (fr), Solomon 19164 (CR, MO); Morales 1328 (INB, MO); Moravia, San Jerónimo, Bajo La Hondura, 11001300 m, 24 may 1976 (fl), Utley \& Utley 5001 (MO); Alajuelita, Z. P. Cerros de Escazú, Cerros Escazú-La Carpintera, $09^{\circ} 51^{\prime} 55^{\prime \prime} \mathrm{N}, 84^{\circ} 08^{\prime} 05^{\prime \prime} \mathrm{W}, 1850-2000 \mathrm{~m}, 17$ ago 1995 (fl), Morales \& Ramírez 4752 (INB, MO); La Palma, 1600 m, 3 feb 1924 (fr), Standley 33154 (US). Guatemala. Quiché: Nebaj, on Chunama, low forest about $13 \mathrm{~km} \mathrm{~N}$ on Cotzal Road, 6000 ft., 1 jul 1964 (fl), Contreras 5143 (MO); Mountain slopes SE of Nebaj along road to El Boquerón, 7000-8000 ft., 10 ago 1964 (fl, fr), Proctor 25467 (MO). Honduras. Francisco Morazán: Cerro La Tigra, near Tegucigalpa, 12 may 1973 (fl), Clewell 3996 (MO); Cerro de Uyuca, near Zamorano, pine brake, 5000-5400 ft., 1 jul 1962 (fl), Webster et al. 11876 (MO); forest near Rancho Quemado, San Juancito mountains, 2200 m, 10 abr 1954 (bo), Williams \& Williams 18902 (US). Olancho: Along Río Olancho, on road between San Francisco de la Paz and Gualaco, $13.6 \mathrm{mi}$ SW of Gualaco, $15^{\circ} 00^{\prime} \mathrm{N}, 86^{\circ} 07^{\prime} \mathrm{W}, 1300 \mathrm{~m}, 6$ feb 1987 (fr), Croat \& Hannon 64219 (MO). Ocotepeque: Belén Gualcho, Cordillera de Celaque, Cruz Alata 3 mi N of Belén Gualcho 


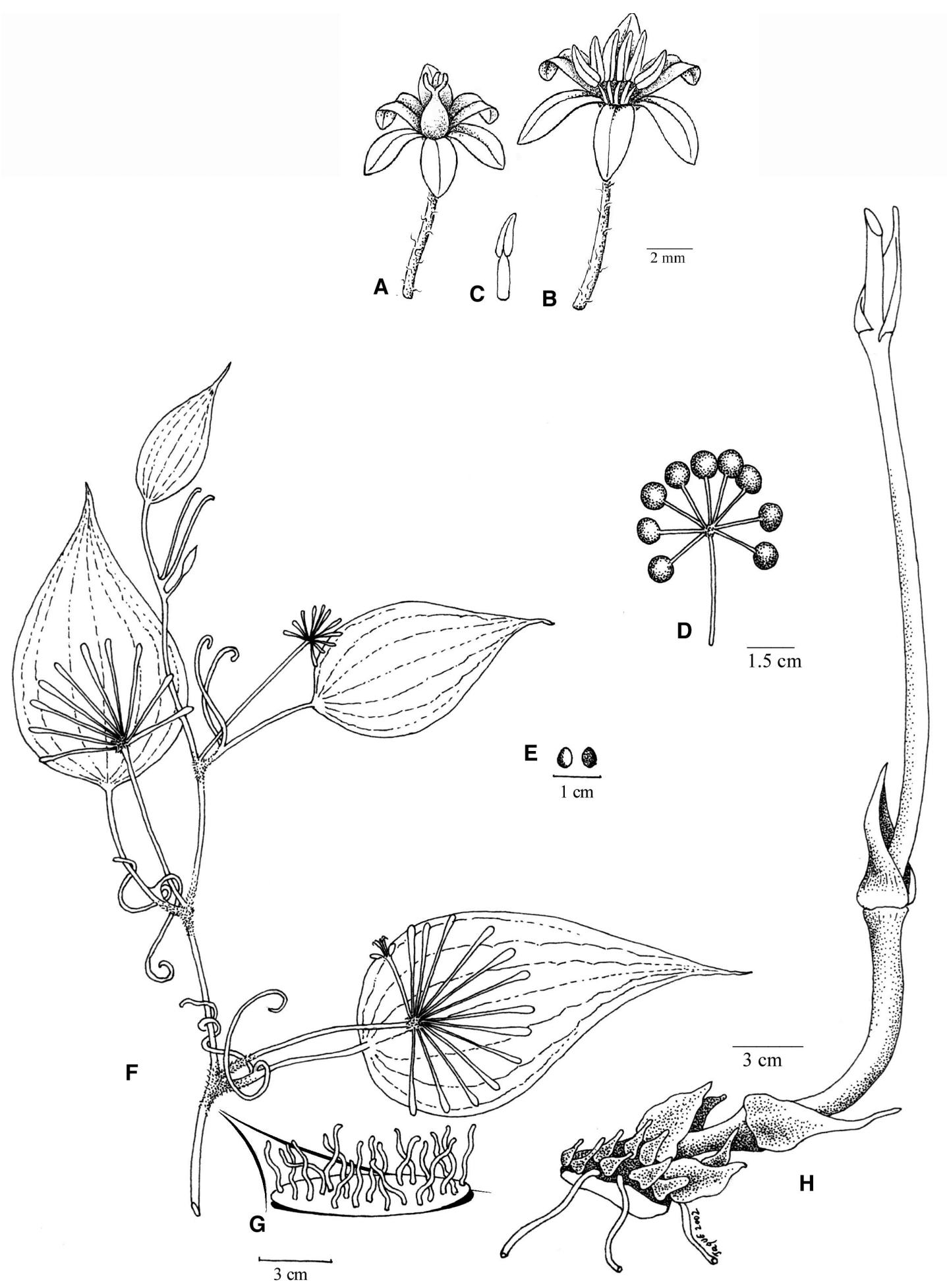

Figura 10. Smilax subpubescens A. DC. A. Flor femenina, B. Flor masculina, C. Estambre, D. Frutos, E. Semillas, F. Rama fértil con flores, G. Tricomas de la base del pecíolo, H. Tallo y rizoma. 

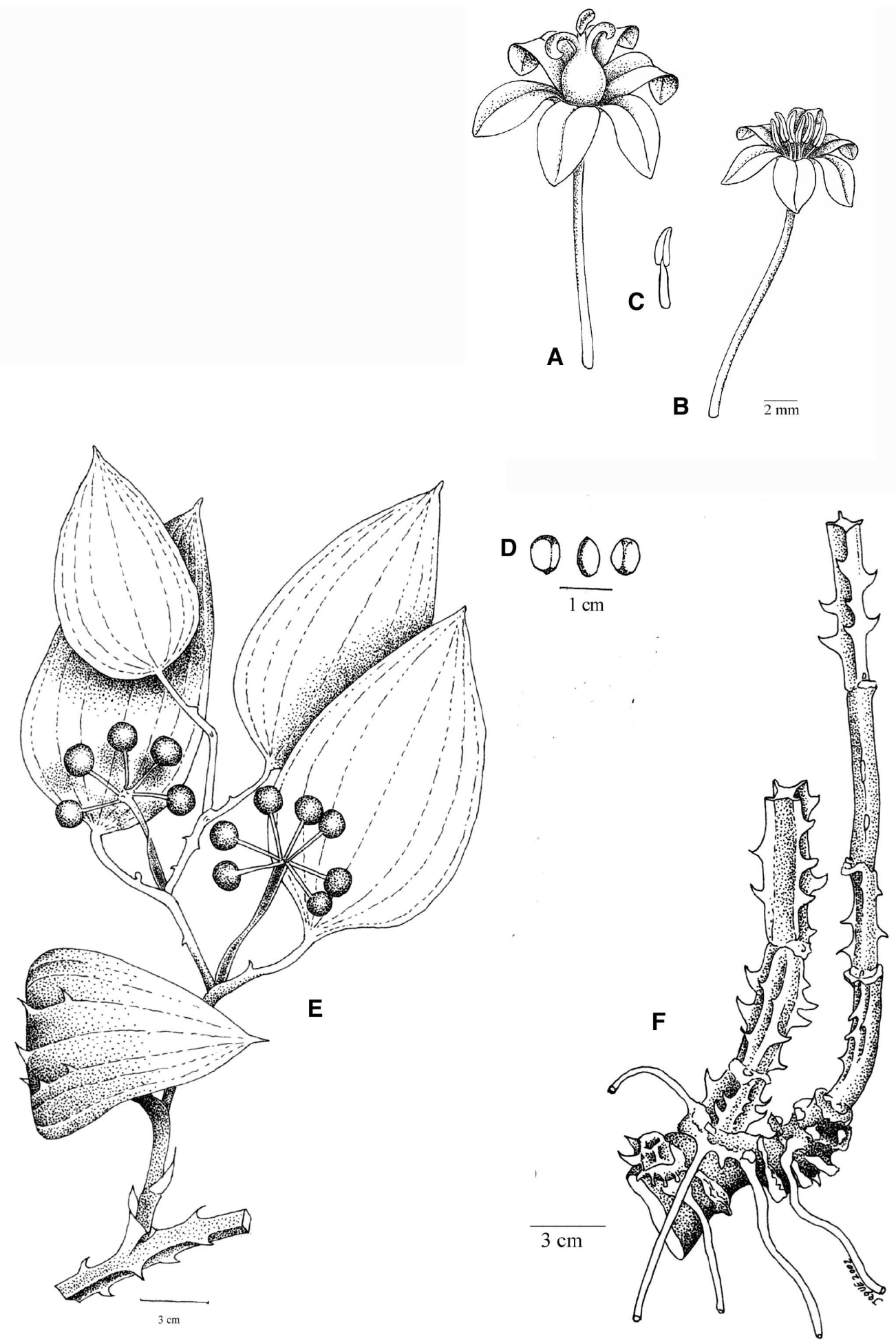

Figura 11. Smilax vanilliodora Apt. A. Flor femenina, B. Flor masculina, C. Estambre, D. Semillas, E. Rama vegetativa con frutos, F. Tallo y rizoma. 
1. La presencia y la ausencia de alas en los tallos y los distintos tamaños de las anteras, en comparación con los filamentos, son caracteres que muchos autores han usado para distinguir $S$. vanilliodora y S. chiriquensis. Los especímenes Gentle 6702 y Sandino 4751 (ambos en MO), que Huft (1994) trata en $S$. regelii, son plantas masculinas con anteras no bien desarrolladas. En el material revisado en Costa Rica se encuentran anteras más cortas o largas que los filamentos en una sola muestra.

2. El color de los frutos varía a lo largo del desarrollo de la maduración. En Costa Rica la mayoría de los especímenes de $S$. vanilliodora recolectados presentan frutos rojos y ocasionalmente son rojos a púrpura. En cambio, en los especímenes de $S$. chiriquensis de Panamá son de color rojo o negro.

3. En la literatura ambos sinónimos han sido descritos con caracteres geográficamente restringidos. Así, S. chiriquensis ha sido tradicionalmente considerada como una especie endémica de Panamá y $S$. vanilliodora endémica de Costa Rica.

4. Killip \& Morton (1936) describen la variedad $S$. regelii var. albida y la distinguen de $S$. regelii var. regelii (excluida aquí, ver adelante) por las bayas

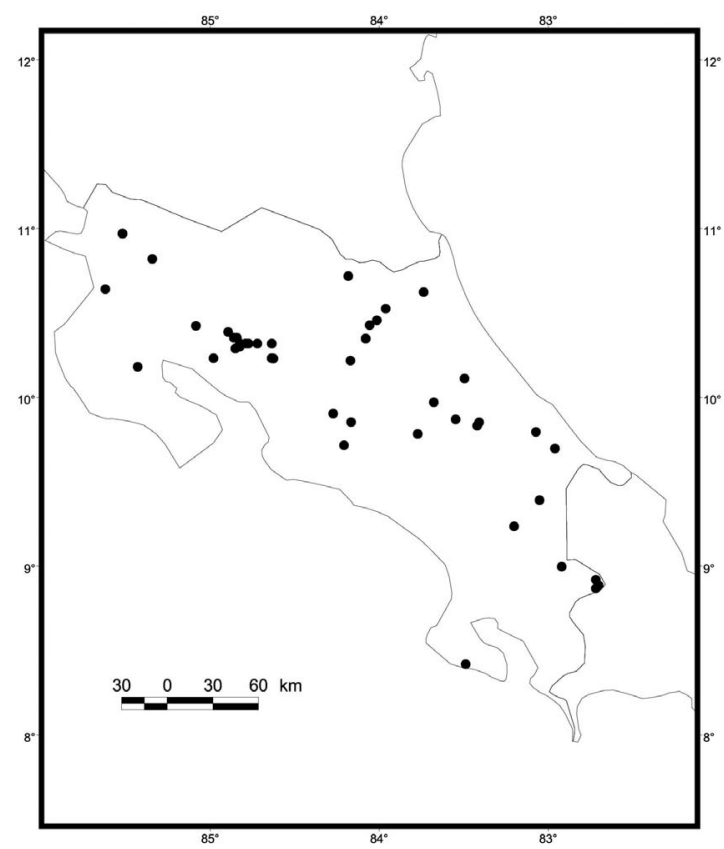

Figura 12. Distribución geográfica de Smilax vanilliodora (•) en Costa Rica. blancas y mencionan que solamente se conoce por el espécimen tipo (Standley 53257, US), que muestra tallos cuadrados con alas poco prominentes, hojas con base cordada y pedúnculos más largos que el pecíolo subyacente, caracteres que coinciden con la descripción de $S$. vanilliodora.

ESPECÍMENES EXAMINADOS. COSTA RICA. Alajuela: Eastern slopes of Volcán Miravalles, west of Bijagua, near the Río Zapote, $10^{\circ} 44^{\prime} \mathrm{N}, 85^{\circ} 5^{\prime} \mathrm{W}, \mathrm{ca} .600 \mathrm{~m}, 11-12$ feb 1982 (fl), Burger et al. 11728 (CR); San Carlos, Cuenca del San Carlos, ca. $7 \mathrm{~km}$ NE Boca Tapada, Lagarto Lodge, $10^{\circ} 41^{\prime} 10^{\prime \prime} \mathrm{N}$, 8410'50"W, 90 m, 27 jul 1996, Hammel 20351 (INB); San Ramón, Reserva Biológica Alberto Manuel Brenes, Cordillera de Tilarán, sendero de entrada a la estación, $10^{\circ} 13^{\prime} 00^{\prime \prime} \mathrm{N}$, 845'00"W, 850 m, 16 mar 1996 (fr), Gómez-Laurito et al. 12847 (USJ); Reserva Biológica Monteverde, Río Peñas Blancas Valley, area near Refugio Alemán, $10^{\circ} 18^{\prime} \mathrm{N}, 84^{\circ} 45^{\prime} \mathrm{W}$, 900-1100 m, 28 feb 1992 (bo, fl), Haber et al. 11297 (INB). Cartago: Tapantí, Forest on ridge between Quebrada Casa Blanca and road to Tránsito, $9^{\circ} 47^{\prime} \mathrm{N}, 83^{\circ} 47^{\prime} \mathrm{W}, c a .1400 \mathrm{~m}, 10$ ago 1984 (fr), Grayum \& Jacobs 3728 (CR, MO); Turrialba, Monumento Nacional Guayabo, Santa Teresita, sobre los Ríos Guayabo, Lajas y Torito, $9^{\circ} 57^{\prime} 50^{\prime \prime} \mathrm{N}, 83^{\circ} 41^{\prime} 30^{\prime \prime} \mathrm{W}, 700-1800$ m, 8 may 1992 (fr), Rivera 1684 (CR); Turrialba, Interamerican Institute of Agricultural Sciences, feb 1950 (bo, fl), León 2277 (USJ); Turrialba, Monumento Nacional Guayabo, 600 m, 1 feb 1900 (fr), Sánchez 53 (USJ). Guanacaste: Liberia, Parque Nacional Guanacaste, Cordillera de Guanacaste, Estación Cacao, Sendero casa de Fran, $10^{\circ} 55^{\prime} 45^{\prime \prime} \mathrm{N}, 8^{\circ} 28^{\prime} 15^{\prime \prime} \mathrm{W}, 1100 \mathrm{~m}, 8$ feb 1995 (bo, fl), Picado 43 (CR, INB, MO); Liberia, Parque Nacional Guanacaste, Cordillera de Guanacaste, Estación Cacao, Sendero Arenales, bosque primario, $10^{\circ} 55^{\prime} 43^{\prime \prime} \mathrm{N}, 85^{\circ} 28^{\prime} 10^{\prime \prime} \mathrm{W}, 1100 \mathrm{~m}, 9$ feb 1995 (fr), Ávila 38 (INB); Rio Chiquito de Tilarán, Rio Negro valley, Atlantic slope, lower montane wet forest, $10^{\circ} 22^{\prime} \mathrm{N} 84^{\circ} 52^{\prime} \mathrm{W}$, 1400 m, 30 jul 1986 (fr), Haber ex Bello 5858 (MO). Heredia: $\mathrm{S}$ base of Cerros Sardinal, Chilamate de Sarapiquí (N side of Río Sarapiquí), $10^{\circ} 27.5^{\prime} \mathrm{N}, 84^{\circ} 04^{\prime} \mathrm{W}, 80-110 \mathrm{~m}, 4$ jul 1985 (fr), Grayum \& Hammel 5545 (MO); Parque Nacional Braulio Carrillo, Los Llanos de Quesada, aluvión del Río Sucio, bosque muy húmedo tropical, 450 m, 11 feb 1984 (fl), Gómez et al. 21018 (CR); Sarapiquí, Puerto Viejo, Finca La Selva, the OTS Field Station on the Río Puerto Viejo just $\mathrm{E}$ of its junction with the Río Sarapiquí, Sendero Ribereño, bluffs above the Río Sarapiquí, 100 m, 5 jun 1985 (fr), Jacobs 3251 (USJ). Limón: Limón, El Progreso, entre Cerro Muchilla y Cerro Avioneta, Fila Matama, siguiendo la fila y los flancos, Valle de la Estrella, 9 $47^{\prime} 40^{\prime \prime} \mathrm{N}, 8^{\circ} 06^{\prime} 30^{\prime \prime} \mathrm{W}, 850 \mathrm{~m}, 8$ abr 1989 (bo, fl), Herrera \& Madrigal 2554 (CR, INB, MO); Pococí, R. N. F. S. Barra del Colorado, Llanura de Tortuguero, Sector Corosí, $10^{\circ} 35^{\prime} 40^{\prime \prime} \mathrm{N}, 8^{\circ} 45^{\prime} 00^{\prime \prime} \mathrm{W}, 100 \mathrm{~m}, 8$ dic 1990 (fr), Rojas 185 (CR); Talamanca, Bratsi, Amubri, Alto Lari, Kivut, $9^{\circ} 24^{\prime} 15^{\prime \prime} \mathrm{N}$, 8305'15"W, 1300 m, 7 mar 1992 (bo, fl), Herrera 5211 (INB). Puntarenas: About $2 \mathrm{~km}$ southeast of Monteverde, on the Pacific watershed, pasture, forest edges, and montane evergreen cloud forest (lower montane wet forest and lower mon- 
tane rain forest zones), $10^{\circ} 18^{\prime} \mathrm{N}, 84^{\circ} 48^{\prime} \mathrm{W}, 1400 \mathrm{~m}, 18-21$ mar 1973 (fl), Burger \& Gentry 8799 (CR, F); Cerro Pando, ridges above the Río Cotón and Río Negro, Southern Puntarenas Prov, 8 ${ }^{\circ} 55^{\prime} \mathrm{N}, 82^{\circ} 45^{\prime} \mathrm{W}, 1000-1800 \mathrm{~m}, 19-21$ feb 1982 (fl), Barringer \& Gómez 1618 (CR); Coto Brus, Fila Cruces, Las Cruces Biological Station, "Melissa Meadow" reforestation area, 1000 m, forest edge, 8 mar 2001 (fr), Werner 226 (USJ); Puntarenas, Cordillera de Tilarán, Monteverde, San Gerardo Biological Station, aging second growth forest premontane we forest, $10^{\circ} 17^{\prime} 0 " \mathrm{~N}, 84^{\circ} 48^{\prime} 00^{\prime \prime} \mathrm{W}, 900 \mathrm{~m}, 8$ mar 1995 (fl), Penneys 214 (CR, INB, MO); Parque Nacional Corcovado, ridge above Río Claro, $8^{\circ} 28^{\prime} \mathrm{N}, 83^{\circ} 35^{\prime} \mathrm{W}, 0-100 \mathrm{~m}, 24$ nov 1981 (fr), Knapp \& Mallet 2198 (MO); Cantón de Puntarenas, Monteverde, Pacific slope, lower montane wet forest, upper community, Campbell farm, $10^{\circ} 18^{\prime} \mathrm{N}, 84^{\circ} 48^{\prime} \mathrm{W}, 1500 \mathrm{~m}, 30$ jul 1991 (fr), Haber \& Zuchowski 10788 (CR, MO). Puntarenas/Alajuela: Evergreen cloud forest and wet wind-gap formations (lower montane and premontane rain forest life zone) on and near the Continental Divide about 2 to $5 \mathrm{~km}$ east and southeast of Monteverde, $10^{\circ} 18^{\prime} \mathrm{N}, 84^{\circ} 46^{\prime} \mathrm{W}, 1580-1700$ m, 17 oct 1978 (fl), Antonio 743 (CR). San José: Acosta, Zona Protectora Cerros de Escazú, Cerros Escazú, La Carpintera, Palmichal, Río Tabarcia, Salvaje, sendero a Cerro Cedral, 9०51'02"N, 8409'52"W, 1500-1800 m, 25 feb 1995 (fr), Morales 3524 (INB); Mora, Zona Protectora El Rodeo, bosque húmedo premontano, bosques de la Universidad para La Paz,

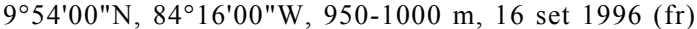
Cascante 1167 (CR); Moravia, Bajo de la Hondura, 1200 m, 8 jun 1977 (fr), Gómez-Laurito \& Bermúdez 2848 (USJ). Guatemala. Petén: La Cumbre, in low forest, east of km 138, bordering village, 29 set 1966 (fr), Contreras 6283 (MO); Cansis, in low forest in corozal, west of Cadenas Road, 13 oct 1966 (fr), Contreras 6418 (MO). Honduras. Atlántida Lancetilla Valley, near Tela, 20-600 m, 6 dec 1927-20 mar 1928 (fr), Standley 52745 (US). Colón: Trujillo, howler site, along Río Selen 7 miles E Trujillo, 19 may 1980, Saunders 285 (MO). MÉXICO. Chiapas: $5 \mathrm{~km}$ al E de Raudales, $130 \mathrm{~m}, 6$ abr 1966 (fl), Quintero 3474 (MO); San Cristóbal de Las Casas, Santa Cruz en San Filipe, 15 nov 1986 (fr), Méndez \& de López 9488 (MO). Veracruz: San Andrés Tuxtla, $2 \mathrm{~km}$ al N de la Estación de Biología Tropical Los Tuxtlas, $18^{\circ} 34^{\prime}$ y $18^{\circ} 36^{\prime} \mathrm{N}$, 9504' y 9509'W, 25 set 1986 (fr), Cedillo 3683 (MO). NicARaguA. Río San Juan: Entre el pueblo de San Juan del Norte Nuevo y la casa de Ramón Castillo viajando por el Caño San Juanillo, $10^{\circ} 55^{\prime} \mathrm{N}, 83^{\circ} 49^{\prime} \mathrm{W}, 0-100$ m, 7 jul 1994, Rueda et al. 1833 (MO). Zelaya: El Zapote, $40 \mathrm{~km}$ al NE de Nueva Guinea, camino al NE de San Martín, $11^{\circ} 49^{\prime} \mathrm{N}, 84^{\circ} 23^{\prime} \mathrm{W}, 130$ 150 m, 26 feb 1984 (bo, fl), Sandino 4751 (MO); Caño Montecristo, al E del campamento Germán Pomares, $11^{\circ} 36^{\prime} \mathrm{N}$, 835' W, 60-90 m, 8 feb 1982 (fr), Moreno 15156 (MO). PANAMÁ. Chiriquí: Vicinity of Fortuna Dam, along trail across valley south of lake forest, $8^{\circ} 45^{\prime} 4^{\prime \prime} \mathrm{N}, 82^{\circ} 15^{\prime} 4^{\prime \prime} \mathrm{W}, 1300-1400$ m, 7 feb 1987 (fr), McPherson 10400 (MO); Bugaba, Santa Clara, Hartmann's Finca, montane forest with distinct dry season, $08^{\circ} 50^{\prime} \mathrm{N} 82^{\circ} 44^{\prime} \mathrm{W}, 1300 \mathrm{~m}, 26$ feb 1985 (fl), van der Werff \& Herrera 7112, 7114 (MO). Panamá: 14 km N of Panamerican Highway on El Llano to Carti Road under canopy, 29 ene 1977 (bo, fl), Folsom \& Collins 1518 (MO).

\section{TAXON EXCLUIDO}

Smilax regelii Killip \& C. Morton var. regelii, Publ. Carnegie Inst. Wash. 461(12): 272. 1936. TIPO: isolectotipo probablemente de "Smilax grandifolia" Regel, ex Horto Bot. Petropolitano” (GH!).

Smilax grandifolia fue descrita por Regel (1856) con base en una planta del Jardín Botánico de San Petersburgo, cuya semilla era originaria de Brasil y fue enviada por Riedel. El tipo no fue encontrado en el Herbario LE de San Petersburgo (Leningrado), Rusia (Andreata 1997). De Candolle (1878) comenta que esta planta fue cultivada en 1875 en el Jardín Botánico de Múnich con un nombre falso y de origen desconocido. Andreata (1997) examinó las colecciones del Herbario M (Múnich), donde halló cinco especímenes con varios nombres, números y diferentes fechas.

Ella considera que el material cultivado en Múnich probablemente fue enviado por Riedel, pero se considera dudoso. Además, podría ser de la Amazonia, donde Riedel estuvo en 1928 (Urban 1906). En el Field Museum de Chicago (F) se encuentra una foto (\# 25119) procedente del Herbario de Ginebra que, al parecer, corresponde a $S$. grandifolia. Andreata (1997) sitúa a $S$. grandifolia como sinónimo de $S$. longifolia y asigna un lectotipo del Herbario de Kew $(\mathrm{K})$, que es un isotipo de LE.

Killip \& Morton (1936) tratan a S. grandifolia Regel como sinónimo de $S$. regelii basados en la descripción hecha por De Candolle (1878), así como en el espécimen del Gray Herbarium (GH), identificado como $S$. grandifolia Regel. Tanto Killip \& Morton como Huft (1994) sugieren que este espécimen es probablemente el material tipo de $S$. grandifolia.

Al examinar el material de $S$. longifolia, de Brasil, muestra tallos con dos hileras de aguijones en cada ángulo, por lo que parece ser una especie diferente a S. vanilliodora.

Agradecimientos. Este estudio es parte de una tesis de maestría en la Escuela de Biología de la Universidad de Costa Rica, con una beca del Servicio Alemán de Intercambio Académico (DAAD) otorgada a la autora. Otras instituciones que hicieron posible esta investigación son el Centro Agronómico Tropical de Investigación y Enseñanza (CATIE) y el Instituto Tecnológico de Costa Rica sede en San Carlos; además la Finca Coope San Juan 
y el Jardín Agroecológico Bougainvillea, con apoyo económico y logístico. El Jardín Botánico de Missouri, a través de una beca Elizabeth Bascom, permitió la revisión de literatura y de especímenes tipo.

\section{LITERATURA CITADA}

Andreata, R.H.P. 1979. Smilax spicata Vell. (Smilacaceae). Considerações taxonômicas. Rodriguesia 31: 105-115.

Andreata, R.H.P. 1980. Smilax Linnaeus (Smilacaceae). Ensaio para uma revisão taxonômica das espécies brasileiras. Arch. Jar. Bot. Rio de Janeiro 24: 179-301.

Andreata, R.H.P. 1982. Smilax L. (Smilacaceae). Espécies brasileiras. II. S. cissoides Mart. ex Griseb. Descricão da flor masculina, nova ocorrência e novo sinônimo. Cadernos Pesq. 2, Sér. Bot. 49: 49-54.

Andreata, R.H.P. 1984a. Smilax L. (Smilacaceae). Espécies brasileiras. I. S. longifolia Richard; localização e classificação dos tipos e seus sinônimos. Rodriguesia 36: 45-50.

Andreata, R.H.P. 1991. Smilacaceae. In: F. de Barros, M. M.R.F. de Melo, S.A.C. Chiea, M. Kirizawa, M.G.L. Wanderley \& S.L. Jung-Mendaçolli (eds.). Flora fanerogâmica da Ilha do Cardoso. Caracterização geral da vegetação e listagem das espécies ocorrentes. São Paulo. Instituto de Botânica 1: 170-171.

Andreata, R.H.P. 1995. Smilacaceae. In: B. Stannard (ed.). Flora of Pico das Almas. Royal Botanic Gardens, Kew. p. 770-772.

Andreata, R.H.P. 1996. Smilacaceae. In: M. P. M. Lima \& R.R. Guedes-Bruni (eds.). Reserva Ecológica de Macaé de Cima, Nova Friburgo, RJ: Aspectos florísticos das espécies vasculares. Jardim Botânico do Rio de Janeiro, Rio de Janeiro, Brasil. p. 433-440.

Andreata, R.H.P. 1997. Revisão das espécies brasileiras do gênero Smilax Linnaeus (Smilacaceae). Pesquisas, Bot. 47: 1-243.

Andreata, R.H.P. \& J. Cowley. 1987. Smilacaceae. In: A.M. Giulietti, N.L. Menezes, J.R. Pirani, M. Meguro \& M.G.L. Wanderley. Flora da Serra do Cipó, Minas Gerais: Caracterização e lista das espécies. Bol. Bot. Univ. São Paulo 9: 132.

Andreata, R.H.P. \& T.S. Pereira. 1990. Morfologia das plântulas de algumas espécies de Smilax. Pesquisas Bot. 41: 7-24.

Andreata, R.H.P. \& M.G.L. Wanderley. 1984b. 195Smilacaceae. In: Flora fanerogâmica do Parque Estadual das Fontes do Ipiranga (São Paulo, Brasil). Hoehnea 11: 114-118.

Apt, F.W. 1922. Beiträge zur Kenntnis der mittelamerikanischen Smilaceen und Sarsaparilldrogen. II. Repert. Spec. Nov. Regni Veg. 18: 407, 416.
Arber, A. 1920. Tendrils of Smilax. Bot. Gaz. 69: 438-422. Arveiller, R. 1985. Notes D’Etymologie et De Lexique. Revue de Lingistique Romane 49:131-134.

Baillon, H. 1894. Smilacaceae. In: Histoire des plantes. Paris, Librairie Hachette 12: 430-433, 530-531.

Bentham, G. \& J.D. Hooker. 1880. Liliaceae. In: Genera Plantarum. London, Lovell Reeve \& Co. 3: 751, 763 764.

Brown, R. 1810. Smilacearum. In: Prodromus Florae Novae Hollandiae. London. p. 292.

Burger, W.C. 1991. Estación Biológica de la Reserva Forestal de San Ramón. In: R. Ortiz (ed.). Memoria de Investigación, Reserva Forestal de San Ramón.San Ramón, Sede de Occidente, Universidad de Costa Rica. p. 11.

Chase, M.W., D.E. Soltis, R.G. Olmstead, D. Morgan, D.H. Les, B.D. Mishler, M.R. Duvall, R.A. Price, H.G. Hills, Y. Qiu, K.A. Kron, J.H. Rettig, E. Conti, J.D. Palmer, J.R. Manhart, K.J. Sytsma, H.J. Michaels, W.J. Kress, K.G. Karol, W.D. Clark, M. Hedrén, B.S. Gaut, R K. Jansen, K. Kim, C.F. Wimpee, J.F. Smith, G.R. Furnier, S.H. Strauss, Q. Xiang, G.M. Plunkett, P.S. Soltis, S.M. Swensen, S.E. Williams, P.A. Gadek, C.J. Quinn, L.E. Eguiarte, E. Golenberg, G.H. Learn jr., S.W. Graham, S.C.H. Barrett, S. Dayanandan \& V.A. Albert. 1993. Phylogenetics of seed plants: an analysis of nucleotide sequences from the plastid gene rbcL. Ann. Missouri Bot. Gard. 80: 528-580.

Chase, M.W., M.R. Duvall, H.G. Hills, J.G. Conran, A.V. Cox, L.E. Eguiarte, J. Hartwell, M.F. Fay, L.R. Caddick, K.M. Cameron \& S. Hoot. 1995a. Molecular phylogenetics of Lilianae. In: P.J. Rudall, P.J. Cribb, D.F. Cutler \& C.J. Humphries (eds.). Monocotyledons: systematics and evolution. Royal Botanic Garden, Kew. p. 109-137.

Chase, M.W., D.W. Stevenson, P. Wilkin \& P.J. Rudall. 1995b. Monocot systematics: a combined analysis. In P.J. Rudall, P.J. Cribb, D.F. Cutler \& C.J. Humphries (eds.). Monocotyledons: systematics and evolution. Royal Botanic Garden, Kew. p. 685-730

Choussy, F. 1926. Flora salvadoreña. Publicación del Ministerio de Instrucción Pública de la República de El Salvador. Tomo I: $\mathrm{p} 1$.

Clos, D. 1857. Les vrilles des Smilax nifolioles ni stipule. Bull. Soc. Bot. Fr. 4: 984-987.

Cronquist, A. 1968. The evolution and classification of flowering plants. Boston, Houghton Mifflin.

Cronquist, A. 1981. An integrated system of classification of flowering plants. Columbia University Press, New York.

Dahlgren, R. \& H. Clifford. 1982. The monocotyledons: A comparative study. London, Academic Press.

Dahlgren, R., H. Clifford \& P. Yeo. 1985. The families of 

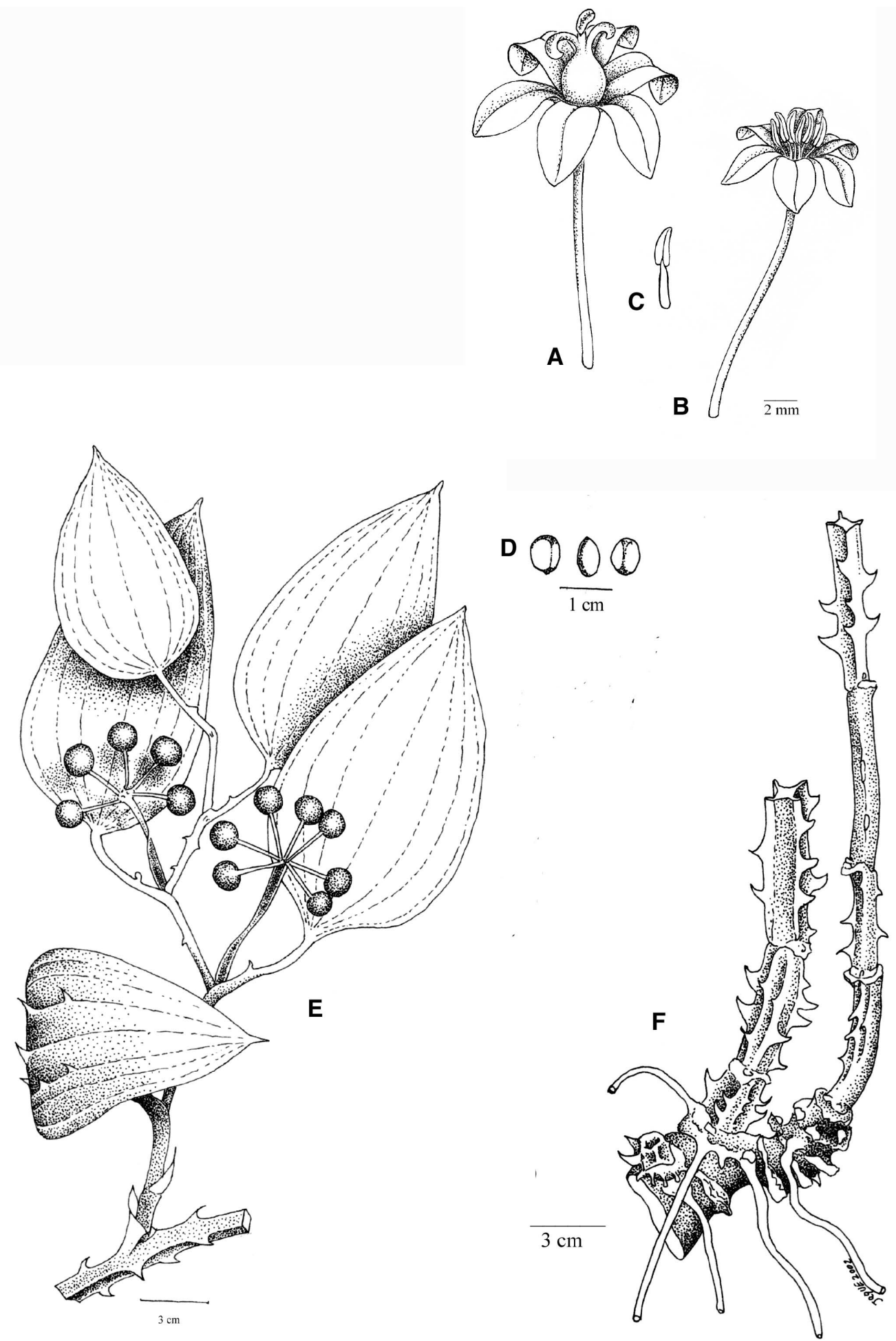

Figura 11. Smilax vanilliodora Apt. A. Flor femenina, B. Flor masculina, C. Estambre, D. Semillas, E. Rama vegetativa con frutos, F. Tallo y rizoma. 
1. La presencia y la ausencia de alas en los tallos y los distintos tamaños de las anteras, en comparación con los filamentos, son caracteres que muchos autores han usado para distinguir $S$. vanilliodora y S. chiriquensis. Los especímenes Gentle 6702 y Sandino 4751 (ambos en MO), que Huft (1994) trata en $S$. regelii, son plantas masculinas con anteras no bien desarrolladas. En el material revisado en Costa Rica se encuentran anteras más cortas o largas que los filamentos en una sola muestra.

2. El color de los frutos varía a lo largo del desarrollo de la maduración. En Costa Rica la mayoría de los especímenes de $S$. vanilliodora recolectados presentan frutos rojos y ocasionalmente son rojos a púrpura. En cambio, en los especímenes de $S$. chiriquensis de Panamá son de color rojo o negro.

3. En la literatura ambos sinónimos han sido descritos con caracteres geográficamente restringidos. Así, S. chiriquensis ha sido tradicionalmente considerada como una especie endémica de Panamá y $S$. vanilliodora endémica de Costa Rica.

4. Killip \& Morton (1936) describen la variedad $S$. regelii var. albida y la distinguen de $S$. regelii var. regelii (excluida aquí, ver adelante) por las bayas

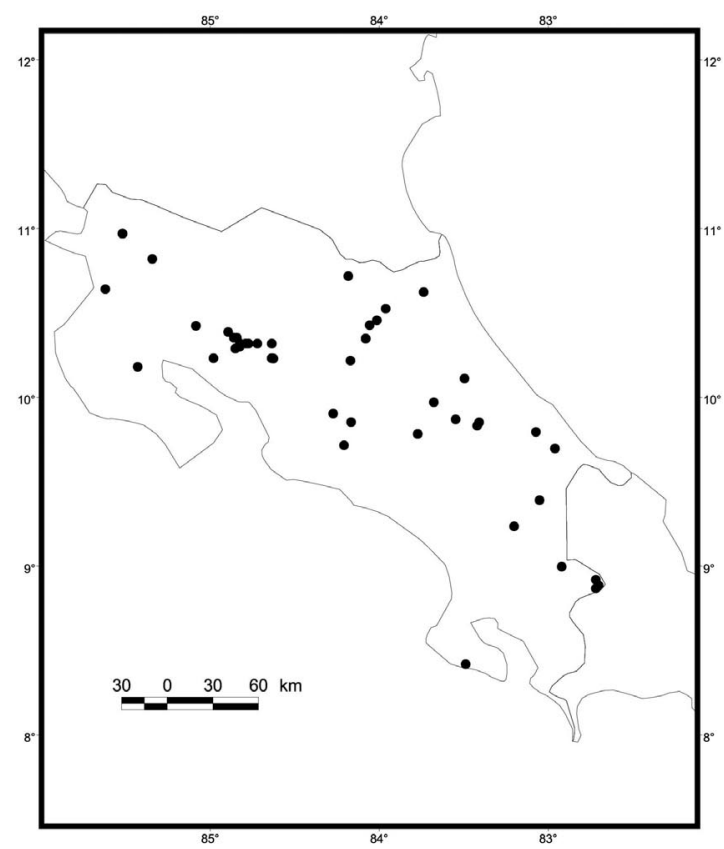

Figura 12. Distribución geográfica de Smilax vanilliodora (•) en Costa Rica. blancas y mencionan que solamente se conoce por el espécimen tipo (Standley 53257, US), que muestra tallos cuadrados con alas poco prominentes, hojas con base cordada y pedúnculos más largos que el pecíolo subyacente, caracteres que coinciden con la descripción de $S$. vanilliodora.

ESPECÍMENES EXAMINADOS. COSTA RICA. Alajuela: Eastern slopes of Volcán Miravalles, west of Bijagua, near the Río Zapote, $10^{\circ} 44^{\prime} \mathrm{N}, 85^{\circ} 5^{\prime} \mathrm{W}, \mathrm{ca} .600 \mathrm{~m}, 11-12$ feb 1982 (fl), Burger et al. 11728 (CR); San Carlos, Cuenca del San Carlos, ca. $7 \mathrm{~km}$ NE Boca Tapada, Lagarto Lodge, $10^{\circ} 41^{\prime} 10^{\prime \prime} \mathrm{N}$, 8410'50"W, 90 m, 27 jul 1996, Hammel 20351 (INB); San Ramón, Reserva Biológica Alberto Manuel Brenes, Cordillera de Tilarán, sendero de entrada a la estación, $10^{\circ} 13^{\prime} 00^{\prime \prime} \mathrm{N}$, 845'00"W, 850 m, 16 mar 1996 (fr), Gómez-Laurito et al. 12847 (USJ); Reserva Biológica Monteverde, Río Peñas Blancas Valley, area near Refugio Alemán, $10^{\circ} 18^{\prime} \mathrm{N}, 84^{\circ} 45^{\prime} \mathrm{W}$, 900-1100 m, 28 feb 1992 (bo, fl), Haber et al. 11297 (INB). Cartago: Tapantí, Forest on ridge between Quebrada Casa Blanca and road to Tránsito, $9^{\circ} 47^{\prime} \mathrm{N}, 83^{\circ} 47^{\prime} \mathrm{W}, c a .1400 \mathrm{~m}, 10$ ago 1984 (fr), Grayum \& Jacobs 3728 (CR, MO); Turrialba, Monumento Nacional Guayabo, Santa Teresita, sobre los Ríos Guayabo, Lajas y Torito, $9^{\circ} 57^{\prime} 50^{\prime \prime} \mathrm{N}, 83^{\circ} 41^{\prime} 30^{\prime \prime} \mathrm{W}, 700-1800$ m, 8 may 1992 (fr), Rivera 1684 (CR); Turrialba, Interamerican Institute of Agricultural Sciences, feb 1950 (bo, fl), León 2277 (USJ); Turrialba, Monumento Nacional Guayabo, 600 m, 1 feb 1900 (fr), Sánchez 53 (USJ). Guanacaste: Liberia, Parque Nacional Guanacaste, Cordillera de Guanacaste, Estación Cacao, Sendero casa de Fran, $10^{\circ} 55^{\prime} 45^{\prime \prime} \mathrm{N}, 8^{\circ} 28^{\prime} 15^{\prime \prime} \mathrm{W}, 1100 \mathrm{~m}, 8$ feb 1995 (bo, fl), Picado 43 (CR, INB, MO); Liberia, Parque Nacional Guanacaste, Cordillera de Guanacaste, Estación Cacao, Sendero Arenales, bosque primario, $10^{\circ} 55^{\prime} 43^{\prime \prime} \mathrm{N}, 85^{\circ} 28^{\prime} 10^{\prime \prime} \mathrm{W}, 1100 \mathrm{~m}, 9$ feb 1995 (fr), Ávila 38 (INB); Rio Chiquito de Tilarán, Rio Negro valley, Atlantic slope, lower montane wet forest, $10^{\circ} 22^{\prime} \mathrm{N} 84^{\circ} 52^{\prime} \mathrm{W}$, 1400 m, 30 jul 1986 (fr), Haber ex Bello 5858 (MO). Heredia: $\mathrm{S}$ base of Cerros Sardinal, Chilamate de Sarapiquí (N side of Río Sarapiquí), $10^{\circ} 27.5^{\prime} \mathrm{N}, 84^{\circ} 04^{\prime} \mathrm{W}, 80-110 \mathrm{~m}, 4$ jul 1985 (fr), Grayum \& Hammel 5545 (MO); Parque Nacional Braulio Carrillo, Los Llanos de Quesada, aluvión del Río Sucio, bosque muy húmedo tropical, 450 m, 11 feb 1984 (fl), Gómez et al. 21018 (CR); Sarapiquí, Puerto Viejo, Finca La Selva, the OTS Field Station on the Río Puerto Viejo just $\mathrm{E}$ of its junction with the Río Sarapiquí, Sendero Ribereño, bluffs above the Río Sarapiquí, 100 m, 5 jun 1985 (fr), Jacobs 3251 (USJ). Limón: Limón, El Progreso, entre Cerro Muchilla y Cerro Avioneta, Fila Matama, siguiendo la fila y los flancos, Valle de la Estrella, 9 $47^{\prime} 40^{\prime \prime} \mathrm{N}, 8^{\circ} 06^{\prime} 30^{\prime \prime} \mathrm{W}, 850 \mathrm{~m}, 8$ abr 1989 (bo, fl), Herrera \& Madrigal 2554 (CR, INB, MO); Pococí, R. N. F. S. Barra del Colorado, Llanura de Tortuguero, Sector Corosí, $10^{\circ} 35^{\prime} 40^{\prime \prime} \mathrm{N}, 8^{\circ} 45^{\prime} 00^{\prime \prime} \mathrm{W}, 100 \mathrm{~m}, 8$ dic 1990 (fr), Rojas 185 (CR); Talamanca, Bratsi, Amubri, Alto Lari, Kivut, $9^{\circ} 24^{\prime} 15^{\prime \prime} \mathrm{N}$, 8305'15"W, 1300 m, 7 mar 1992 (bo, fl), Herrera 5211 (INB). Puntarenas: About $2 \mathrm{~km}$ southeast of Monteverde, on the Pacific watershed, pasture, forest edges, and montane evergreen cloud forest (lower montane wet forest and lower mon- 